\title{
Slit maps in the study of equal-strength cavities in $n$-connected elastic planar domains
}

\author{
Y.A. AntiPov \\ Department of Mathematics, Louisiana State University \\ Baton Rouge LA 70803, USA
}

\begin{abstract}
The inverse problem of plane elasticity on $n$ equal-strength cavities in a plane subjected to constant loading at infinity and in the cavities boundary is analyzed. By reducing the governing boundary value problem to the Riemann-Hilbert problem on a symmetric Riemann surface of genus $n-1$ a family of conformal mappings from a parametric slit domain onto the $n$-connected elastic domain is constructed. The conformal mappings are presented in terms of hyperelliptic integrals and the zeros of the first derivative of the mappings are analyzed. It is shown that for any $n \geq 1$ there always exists a set of the loading parameters for which these zeros generate inadmissible poles of the solution.
\end{abstract}

\section{Introduction}

Analysis of the stresses induced by the presence of inclusions and cavities in an elastic matrix subjected to loading have been of interest for over a century [10, [13, [9]. Inverse problems of elasticity which concern problems of determination of the shapes of curvilinear inclusions and cavities with prescribed properties excite particular attention due to their relevance to the material design [11, [3, 17], 2], 15]. A considerable amount of work examines equal-strain inclusions subjected to uniform loading with uniform distribution of stresses inside. By analyzing the stress distribution in composites with single elliptic and ellipsoidal inclusions in two- and three-dimensional unbounded elastic bodies Eshelby [5] established that the stress fields are uniform in the interior of the inclusions provided the matrix is loaded uniformly at infinity. He also conjectured that there do not exist other shapes of single inclusions with such a property. This conjecture was proved for the plane and anti-plane model problem in the simply-connected case in [14. An alternative proof for the antiplane case by the method of conformal mappings was proposed in [12].

Motivated by the problem of designing perforated structures of minimum weight Cherepanov [3] studied the inverse problem of elasticity on a plane uniformly loaded at infinity and having $n$ holes. The boundary of the holes is subjected to constant normal and tangential traction, and the holes profile $L_{j}(j=0,1, \ldots, n-1)$ are determined from an extra boundary condition. It states that the tangential normal stress $\sigma_{t}$ is the same constant, $\sigma$, in all the contours $L_{j}$. For the solution, a conformal map of an $n$-connected slit domain $\mathcal{D}^{e}$ into the elastic domain $D^{e}$, the exterior of the $n$ holes, is applied. The map transforms the boundary value problem into two Schwarz problems of the theory of analytic functions on the $n$ slits. The feature of the map $z=\omega(\zeta)$ employed [3] is that it maps the point $\zeta=\infty$ into the point $z=\infty$, and the exterior of $n$ parallel slits of the parametric plane into the $n$-connected domain $D^{e}$. In general, unless $n \leq 3$, for such a map these slits do not lie in the same line. Cherepanov [3] solved by quadratures the problem in the simply and doubly connected symmetric cases and also analyzed the periodic and doubly periodic problems. Vigdergauz [17] noticed and corrected an error in the computations [3] implemented for the symmetric doubly connected case when the slit domain is the exterior of the cuts $[-2,-1]$, 
$[1,2]$, and the map $z=\omega(\zeta)$ has the property $\omega: \infty \rightarrow \infty$. For the symmetric case [3], the loading parameters $a$ and $b$ are real, and the two equal-strength holes exist if $|b / a|<1$ [17].

To solve the Cherepanov problem for any $n$-connected domain, Vigdergauz [17] proposed to employ a circular map from the exterior of $n$-circles onto the $n$-connected elastic domain. Application of the Sherman integral representation reduced the resulting boundary value problem to integral equations solved numerically by the method of least squares. This method was further developed in [18 for doubly periodic structures by using an integral representation with quasi-automorphic analogues of the Cauchy kernel and the numerical method of least squares for solving the governing integral equations. An alternative explicit representation in terms of the Weierstrass elliptic function for the profile of an inclusion in the case of a doubly periodic structure was given in [6]. The antiplane shear problem for two equal-strain inclusions by means of the Weierstrass zeta function was treated in [7].

The main goal of this paper was to derive a closed-form representation of a family of conformal mappings $z=\omega(\zeta), \omega: \mathcal{D}^{e} \rightarrow D^{e}$ for $n=2$ in the non symmetric case and for $n \geq 3$ and therefore to determine a family of the profiles of $n$ equal-strength holes. In addition, we aim to derive necessary and sufficient conditions for the solution to exist. This will be achieved by studying the poles of one of the Kolosov-Muskhelishvili complex potentials due to possible zeros of the derivative $\omega^{\prime}(\zeta)$ of the conformal mapping. The stress field $\left\{\sigma_{1}, \sigma_{2}, \tau_{12}\right\}$ is expressible through two functions $\Phi$ and $\Psi$ [10] which have to be analytic everywhere in the domain $D^{e}$. One of them, $\Phi(z)$, is a constant, while the second one has the form [3], p. 918

$$
\Psi(\omega(\zeta))=\frac{F_{+}(\zeta)+F_{-}(\zeta)}{2 \omega^{\prime}(\zeta)}, \quad \zeta \in \mathcal{D}^{e}=\mathbb{C} \backslash l,
$$

where $F_{ \pm}(\zeta)$, the solutions to certain Schwarz problems, are analytic functions in $\mathbb{C} \backslash l$, and $l$ is the union of the slits $l_{j}, j=0,1, \ldots, n-1$, in the parametric $\zeta$-plane. If $\omega^{\prime}(\zeta)$ has zeros in the slit domain $\mathcal{D}^{e}$, then the potential $\Psi(z)$ has inadmissible poles at the images of these zeros. In [20, under the assumption that the solution $\Psi(z)$ found is analytic in the exterior of $n$ holes by applying the maximum principle it was shown that the condition $\left|\left(\sigma_{2}^{\infty}-\sigma_{1}^{\infty}\right) /\left(\sigma_{2}^{\infty}+\sigma_{1}^{\infty}\right)\right| \leq 1$ is necessary for the existence of the solution. Here, $\sigma_{1}^{\infty}$ and $\sigma_{2}^{\infty}$ are constant stresses applied at infinity. To the author's knowledge, no sufficient solvability conditions for the case $n \geq 3$ and for two nonsymmetric cavities and associated exact representations for equal-strength cavities profiles are available in the literature.

In Section 2, we formulate the problem as two Schwarz problems for two auxiliary functions coupled by two conditions. These conditions guarantee that the potential $\Psi$ is analytic everywhere in the domain $D^{e}$ and that the conformal map is single valued. Section 3 gives an integral representation in terms of elliptic integrals of the mapping function for $n=2$ in the general not necessarily symmetric case. The map has two free parameters and, in addition, has two free scaling parameters. It is shown that if $\gamma=|b / a|<1$, then the solution always exists. Here,

$$
a=\frac{1}{2}(\sigma-p)+i \tau, \quad b=\frac{1}{2}\left(\sigma_{2}^{\infty}-\sigma_{1}^{\infty}\right)+i \tau^{\infty},
$$

$\sigma_{1}^{\infty}, \sigma_{2}^{\infty}$, and $\tau^{\infty}$ are constant stresses applied at infinity, $p$ and $\tau$ are the traction components applied to the holes boundaries, and $\sigma=\sigma_{1}^{\infty}+\sigma_{2}^{\infty}-p$. If $\gamma>1$, then the function $\Psi(\zeta)$ has four poles, while the contours $L_{1}$ and $L_{2}$, the profiles of the holes, may (for sufficiently large values of the parameter $\gamma$ ) or may not intersect. If $\gamma=1$, then the contours are two straight segments, and the function $\Psi(z)$ has removable singularities on the contours. In Section 4, we analyze the triply connected case. To pursue the goal to describe the general family of equal-strength cavities, we construct the most general form of the conformal map possible in the case $n=3$. It maps the exterior of three slits $[-1 / k,-1],\left[k_{1}, k_{2}\right]$, and $[1,1 / k]\left(0<k<1,-1<k_{1}<k_{2}<1\right)$ into the triply 




Figure 1: Geometry of the problem

connected domain $D^{e}$, while the infinite point $z=\infty$ is the image of a point $\zeta_{\infty}=\zeta_{\infty}^{\prime}+i \zeta_{\infty}^{\prime \prime}$. Thus, in addition to two scaling parameters it has five real free parameters, $k, k_{1}, k_{2}, \zeta_{\infty}^{\prime}$, and $\zeta_{\infty}^{\prime \prime}$. The governing boundary value problem reduces to a symmetric Riemann-Hilbert problem on a genus- 2 Riemann surface and similarly to [1] is solved exactly. It is shown that if $\zeta_{\infty}$ is a finite point, then regardless of the values of the parameter $\gamma$ the function $\Psi(z)$ has always inadmissible poles in $D^{e}$, and the solution does not exist, that is the condition $\gamma<1$ is necessary but not sufficient. In Section 5 , we identify a family of $n$-connected domains which can be interpreted as images of a slit domain with cuts locating in the same line. We choose the parametric domain $\mathcal{D}^{e}$ as the union of slits in the real axis and assume that $\omega: \infty \rightarrow \infty$. The conformal map with such properties has $2 n-2$ free real parameters. In particular, for the case $n=3$ and $\zeta_{\infty}=\infty$, we show that the family of conformal mappings is four-parametric (the slits are $\left[-1, k_{1}\right],\left[k_{2}, k_{3}\right]$, and $\left[k_{4}, 1\right]$ ), and if $\gamma<1$, then the solution is free of poles. Otherwise, if $\gamma>1$, it has six poles, and the solution does not exist. In Appendix we analyze the case $n=1$ and show that the potential $\Psi(z)$ has two inadmissible poles if $\gamma>1$. The same solvability condition for the case $n=1$ by a different method was derived earlier in [19].

\section{Formulation}

Consider the following problem of plane elasticity [3] (Figure 1).

Let an infinite isotropic plane subjected to constant stresses at infinity, $\sigma_{1}=\sigma_{1}^{\infty}, \sigma_{2}=\sigma_{2}^{\infty}$, and $\tau_{12}=\tau^{\infty}$, have $n$ holes $D_{0}, D_{1}, \ldots D_{n-1}$. Assume that constant normal and tangential traction components are applied to their boundaries $L_{j}, \sigma_{n}=p, \tau_{n t}=\tau, j=0,1, \ldots, n-1$. Find the shape and location of the holes such that the tangent normal stress $\sigma_{t}$ is constant, $\sigma_{t}=\sigma$, in all the contours $L_{j}$.

Let $\Phi(z)$ and $\Psi(z)\left(z=x_{1}+i x_{2}\right)$ be the Kolosov-Muskhelishvili potentials of the problem. These functions are analytic everywhere in the $n$-connected domain $D^{e}=\mathbb{C} \backslash D, D=\cup_{j=0}^{n-1} D_{j}$ and continuous in $D^{e}$ up to its boundary. The equilibrium equations of plane elasticity are satisfied if the stresses are [10]

$$
\sigma_{1}+\sigma_{2}=4 \operatorname{Re} \Phi(z), \quad \sigma_{2}-\sigma_{1}+2 i \tau_{12}=2\left[\bar{z} \Phi^{\prime}(z)+\Psi(z)\right] .
$$

The stresses and the traction vector components on the boundaries are connected by the relations

$$
\sigma_{t}+\sigma_{n}=\sigma_{1}+\sigma_{2}, \quad \sigma_{t}-\sigma_{n}+2 i \tau_{n t}=e^{2 i \alpha(z)}\left(\sigma_{2}-\sigma_{1}+2 i \tau_{12}\right) .
$$


Here, $\alpha(z)$ is the angle between the positive direction of the $x_{1}$-axis and the external normal $n$ to the cavity boundary (internal with respect to the body $\left.D^{e}\right)$. At infinity, the functions $\Phi(z)$ and $\Psi(z)$ behave as 10 ]

$$
\begin{aligned}
& \Phi(z)=b^{\prime}+\frac{X+i Y}{2 \pi(1+\kappa)} \frac{1}{z}+O\left(\frac{1}{z^{2}}\right), \\
& \Psi(z)=b-\frac{\kappa(X-i Y)}{2 \pi(1+\kappa)} \frac{1}{z}+O\left(\frac{1}{z^{2}}\right),
\end{aligned}
$$

where

$$
\begin{gathered}
b^{\prime}=\frac{\sigma_{1}^{\infty}+\sigma_{2}^{\infty}}{4}+i C^{\prime}, \quad b=\frac{1}{2}\left(\sigma_{2}^{\infty}-\sigma_{1}^{\infty}\right)+i \tau^{\infty}, \quad \kappa=\frac{\lambda+3 \mu}{\lambda+\mu}, \\
X+i Y=\sum_{j=0}^{n-1}\left(X_{j}+i Y_{j}\right), \quad X_{j}+i Y_{j}=\int_{L_{j}}\left(X_{j n}+i Y_{j n}\right) d s,
\end{gathered}
$$

$\lambda$ and $\mu$ are the Lamé constants, $\left(X_{j}, Y_{j}\right)$ is the total force applied to the boundary $L_{j}(j=$ $0, \ldots, n-1)$ from the side of a normal directed towards the body $D^{e}$, and $X_{j n}$ and $Y_{j n}$ are the $x_{1^{-}}$ and $x_{2}$-projections of the force at a point of the boundary. Since the traction components $\sigma_{n}$ and $\tau_{n t}$ are constant on the boundary, $X=Y=0$.

The boundary condition $\operatorname{Re} \Phi(z)=\frac{1}{4}\left(\sigma_{1}^{\infty}+\sigma_{2}^{\infty}\right)$ on $L_{j}, j=0, \ldots, n-1$, and the condition at infinity (2.3) imply that the function $\Phi(z)$ is a constant everywhere in $D^{e}, \Phi(z)=\frac{1}{4}\left(\sigma_{1}^{\infty}+\sigma_{2}^{\infty}\right)+i C^{\prime}$. The constant $C^{\prime}$ does not affect the stresses. It is expressed through the rotation $\varepsilon_{\infty}$ of the plane at infinity, $C^{\prime}=2 \mu(1+\kappa)^{-1} \varepsilon_{\infty}[10$.

Due to (2.1), (2.2), (2.3) the analytic function $\Psi(z)$ has to satisfy the conditions

$$
\Psi(z)=a e^{-2 i \alpha(z)}, \quad z \in L=\cup_{j=0}^{n-1} L_{j}, \quad \Psi(z)=b+O\left(z^{-2}\right), \quad z \rightarrow \infty, \quad a=\frac{1}{2}(\sigma-p)+i \tau .
$$

It is known [4], 8] that there exists an analytic function $z=\omega(\zeta)$ that conformally maps the extended complex $\zeta$-plane $\mathbb{C} \cup \infty$ cut along $n$ segments parallel to the real $\zeta$-axis onto the $n$ connected domain $D^{e}$ in the $z$-plane. Such a function is a one-to-one map. The infinite point $z=\infty$ is the image of a certain point $\zeta=\zeta_{\infty}$, and in the vicinity of that point, the conformal map $\omega(\zeta)$ can be represented as

$$
\omega(\zeta)=\frac{c_{-1}}{\zeta-\zeta_{\infty}}+c_{0}+\sum_{j=1}^{\infty} c_{j}\left(\zeta-\zeta_{\infty}\right)^{j}
$$

If $\zeta_{\infty}=\infty$, then

$$
\omega(\zeta)=c_{-1} \zeta+c_{0}+\sum_{j=1}^{\infty} \frac{c_{j}}{\zeta^{j}} .
$$

The boundary condition in (2.5) written in the $\zeta$-plane reads [3]

$$
\psi(\zeta) \omega^{\prime}(\zeta)+a \overline{\omega^{\prime}(\zeta)}=0, \quad \zeta \in l=\cup_{j=0}^{n-1} l_{j},
$$

where $\psi(\zeta)=\Psi(z)$. The problem is significantly simplified for the two analytic functions

$$
F_{ \pm}(\zeta)=[\psi(\zeta) \pm \bar{a}] \omega^{\prime}(\zeta)
$$

The new functions solve the following boundary value problem.

Find two functions $F_{ \pm}(\zeta)$ analytic in the domain $\mathbb{C} \backslash l$ continues up to the boundary $l$, having at most integrable singularities at the endpoints of the contour $l$ and satisfying the Schwarz boundary conditions on the cuts $l$

$$
\operatorname{Re} F_{+}(\zeta)=0, \quad \operatorname{Im} F_{-}(\zeta)=0, \quad \zeta \in l .
$$


If $\zeta_{\infty}$ is a finite point, then the functions $F_{ \pm}(\zeta)$ behave at $\zeta_{\infty}$ and $\infty$ as

$$
F_{ \pm}(\zeta)=-\frac{c_{-1}(b \pm \bar{a})}{\left(\zeta-\zeta_{\infty}\right)^{2}}+O(1), \quad \zeta \rightarrow \zeta_{\infty}, \quad F_{ \pm}(\zeta)=O\left(\zeta^{-2}\right), \quad \zeta \rightarrow \infty .
$$

In the case $\zeta_{\infty}=\infty$,

$$
F_{ \pm}(\zeta)=c_{-1}(b \pm \bar{a})+O\left(\zeta^{-2}\right), \quad \zeta \rightarrow \infty .
$$

The two Schwarz problems are coupled by the conditions

(i) the difference $F_{+}(\zeta)-F_{-}(\zeta)$ may not have zeros in the domain $\mathbb{C} \backslash l$;

(ii) the following $n$ integrals over the loops $l_{j}$ vanish:

$$
\int_{l_{j}}\left[F_{+}(\zeta)-F_{-}(\zeta)\right] d \zeta=0, \quad j=0,1, \ldots n-1
$$

If the two functions $F_{+}(\zeta)$ and $F_{-}(\zeta)$ are known, then the functions $\omega^{\prime}(\zeta)$ and $\psi(\zeta)$ are determined by

$$
\omega^{\prime}(\zeta)=\frac{F_{+}(\zeta)-F_{-}(\zeta)}{2 \bar{a}}, \quad \psi(\zeta)=\frac{F_{+}(\zeta)+F_{-}(\zeta)}{2 \omega^{\prime}(\zeta)}, \quad \zeta \in \mathbb{C} \backslash l .
$$

The condition (i) is necessary and sufficient for the function $\psi(\zeta)$ to be analytic in the domain $\mathbb{C} \backslash l$, while the condition (ii) guarantees that the function $\omega(\zeta)$ is single-valued.

To justify the relations (2.11) in the case $\left|\zeta_{\infty}\right|<\infty$, we note that $\zeta=\infty$ is the $\omega$-image of a finite point of the domain $D^{e}$, the function $\psi(\zeta)$ is bounded at infinity and also

$$
\omega(\zeta)=d_{0}+\frac{d_{1}}{\zeta}+\frac{d_{2}}{\zeta^{2}}+\ldots, \quad \omega^{\prime}(\zeta)=-\frac{d_{1}}{\zeta^{2}}-\frac{2 d_{2}}{\zeta^{3}}-\ldots, \quad \zeta \rightarrow \infty .
$$

In a neighborhood of the point $\zeta_{\infty} \in(-1,1)$ the function $\omega(\zeta)$ admits the expansion (2.6) and therefore

$$
\psi(\zeta)=b+O\left(\left(\zeta-\zeta_{\infty}\right)^{2}\right), \quad \omega^{\prime}(\zeta)=-\frac{c_{-1}}{\left(\zeta-\zeta_{\infty}\right)^{2}}+c_{1}+2 c_{2}\left(\zeta-\zeta_{\infty}\right)+\ldots, \quad \zeta \rightarrow \zeta_{\infty}
$$

\section{Two cavities}

\subsection{Two cavities when $\zeta_{\infty}$ is a finite point: the general case}

Every doubly connected domain $D^{e}$ may be conformally mapped by a function $\zeta=\omega^{-1}(z)$ onto a slit domain $\mathcal{D}^{e}$, the extended complex $\zeta$-plane cut along the two cuts $l_{0}=[-1 / k,-1]$ and $l_{1}=[1,1 / k]$, where $k \in(0,1)$. Moreover, it is possible to choose the map such that the infinite point $z=\infty$ falls into a point $\zeta_{\infty}$ in the open segment $(-1,1)$ of the real $\zeta$-axis. Such a map can be expressed through elliptic integrals.

We introduce an elliptic surface $\mathcal{R}$ of the algebraic function $u^{2}=p_{2}(\zeta)$, where $p_{2}(\zeta)=\left(\zeta^{2}-\right.$ 1) $\left(\zeta^{2}-1 / k^{2}\right)$. A single branch $f(\zeta)$ of the function $p_{2}^{1 / 2}(\zeta)$ is fixed in the $\zeta$-plane cut along the two segments $l_{0}$ and $l_{1}$ by the condition $p_{2}^{1 / 2}(\zeta) \sim \zeta^{2}, \zeta \rightarrow \infty$. This branch is pure imaginary on the sides of the cuts, $f^{ \pm}(\zeta)=\mp i(-1)^{j} \sqrt{\left|p_{2}(\zeta)\right|}, \zeta \in l_{j}, j=0,1$, and real for $\zeta=\xi$ lying outside the cuts in the real axis, $f(\xi)>0,|\xi|>1 / k$, and $f(\xi)<0,-1<\xi<1$.

Since $\operatorname{Re} F_{+}(\zeta)=0$ and $\operatorname{Im} F_{-}(\zeta)=0$ on $l$, the functions $i F_{+}(\zeta)$ and $F_{-}(\zeta)$ can analytically and symmetrically be continued onto the whole Riemann surface. The new functions, $F_{1}(\zeta, u)$ and $F_{2}(\zeta, u)$, given by

$$
F_{1}(\zeta, u)=\left\{\begin{array}{rl}
i F_{+}(\zeta), & (\zeta, u) \in \mathbb{C}_{1}, \\
-i \overline{F_{+}(\bar{\zeta}),}, & (\zeta, u) \in \mathbb{C}_{2},
\end{array} \quad F_{2}(\zeta, u)= \begin{cases}F_{-}(\zeta), & (\zeta, u) \in \mathbb{C}_{1} \\
\overline{F_{-}(\bar{\zeta})}, & (\zeta, u) \in \mathbb{C}_{2}\end{cases}\right.
$$


are rational on the surface and satisfy the symmetry condition

$$
\overline{F_{j}\left(\zeta_{*}, u_{*}\right)}=F_{j}(\zeta, u), \quad(\zeta, u) \in \mathcal{R},
$$

where $\left(\zeta_{*}, u_{*}\right)=(\bar{\zeta},-u(\bar{\zeta}))$ is the point symmetrical to the point $(\zeta, u)$ with respect to the line along which the two sheets $\mathbb{C}_{1}$ and $\mathbb{C}_{2}$ of the surface are connected. Note that if $(\zeta, u) \in \mathbb{C}_{1}$, then $\left(\zeta_{*}, u_{*}\right) \in \mathbb{C}_{2}$.

At the points $\left(\zeta_{\infty}, u_{\infty}\right) \in \mathbb{C}_{1}$ and $\left(\zeta_{\infty}^{*}, u_{\infty}^{*}\right) \in \mathbb{C}_{2}\left(\zeta_{\infty}^{*}=\bar{\zeta}_{\infty}\right)$, both of the functions $F_{1}(\zeta, u)$ and $F_{2}(\zeta, u)$ have order-2 poles with zero residues. At the branch points of the surface, they have simple poles (in the sense of Riemann surfaces). At the two infinite points of the surface they have order-2 zeros. The Schwarz boundary conditions (2.10) can be rewritten as the two symmetric Riemann-Hilbert problems on the elliptic surface $\mathcal{R}$

$$
F_{j}^{+}(\xi, u)-F_{j}^{-}(\xi)=0, \quad \xi \in l=l_{0} \cup l_{1} .
$$

The solutions of these problems are rational function in the surface $\mathcal{R}$. When written in the first sheet, they have the form

$$
\begin{gathered}
F_{-}(\zeta)=\frac{1}{\left(\zeta-\zeta_{\infty}\right)^{2}}\left(A_{0}^{-}+\frac{i\left(A_{1}^{-}+A_{2}^{-} \zeta+A_{3}^{-} \zeta^{2}\right)}{f(\zeta)}\right), \quad \zeta \in \mathbb{C} \backslash l, \\
F_{+}(\zeta)=\frac{1}{\left(\zeta-\zeta_{\infty}\right)^{2}}\left(i A_{0}^{+}+\frac{A_{1}^{+}+A_{2}^{+} \zeta+A_{3}^{+} \zeta^{2}}{f(\zeta)}\right), \quad \zeta \in \mathbb{C} \backslash l,
\end{gathered}
$$

where $A_{j}^{ \pm}$are real constants to be determined. Denote

$$
c_{-1}=c^{\prime}+i c^{\prime \prime}, \quad b \pm \bar{a}=\alpha^{ \pm}+i \beta^{ \pm} .
$$

Due to (2.5) the four parameters $\alpha^{ \pm}$and $\beta^{ \pm}$are expressed through the loading data as

$$
\alpha^{+}=\sigma_{2}^{\infty}-p, \quad \alpha^{-}=p-\sigma_{1}^{\infty}, \quad \beta^{+}=\tau^{\infty}-\tau, \quad \beta^{-}=\tau^{\infty}+\tau .
$$

On expanding the functions (3.4) in a neighborhood of the point $\zeta=\zeta_{\infty}$ and satisfying the first condition in (2.11) we determine $A_{0}^{ \pm}$

$$
A_{0}^{+}=-c^{\prime} \beta^{+}-c^{\prime \prime} \alpha^{+}, \quad A_{0}^{-}=-c^{\prime} \alpha^{-}+c^{\prime \prime} \beta^{-},
$$

and derive four real equations for the other six coefficients

$$
\begin{aligned}
& A_{1}^{ \pm}+\zeta_{\infty} A_{2}^{ \pm}+\zeta_{\infty}^{2} A_{3}^{ \pm}=d^{ \pm}, \\
& A_{2}^{ \pm}+2 \zeta_{\infty} A_{3}^{ \pm}=\frac{d^{ \pm} p_{2}^{\prime}\left(\zeta_{\infty}\right)}{2 p_{2}\left(\zeta_{\infty}\right)},
\end{aligned}
$$

where

$$
d^{+}=\sqrt{\left|p_{2}\left(\zeta_{\infty}\right)\right|}\left(c^{\prime} \alpha^{+}-c^{\prime \prime} \beta^{+}\right), \quad d^{-}=\sqrt{\left|p_{2}\left(\zeta_{\infty}\right)\right|}\left(c^{\prime} \beta^{-}+c^{\prime \prime} \alpha^{-}\right) .
$$

Denoting

$$
A_{0}=i A_{0}^{+}-A_{0}^{-}, \quad A_{j}=A_{j}^{+}-i A_{j}^{-}, \quad j=1,2,3,
$$

and applying formula (2.14) we obtain the derivative of the conformal map

$$
\omega^{\prime}(\zeta)=\frac{1}{2 \bar{a}\left(\zeta-\zeta_{\infty}\right)^{2}}\left(A_{0}+\frac{A_{1}+A_{2} \zeta+A_{3} \zeta^{2}}{f(\zeta)}\right)
$$


In general, the map $z=\omega(\zeta)$ given by (3.11) is a multi-valued function. It is a one-to-one map if

$$
\int_{l_{0}} \omega^{\prime}(\zeta) d \zeta=0, \quad \int_{l_{1}} \omega^{\prime}(\zeta) d \zeta=0
$$

The two integrals over the loops $l_{0}$ and $l_{1}$ vanish if the coefficients $A_{j}^{ \pm}(j=1,2,3)$ solve the four equations

$$
\begin{aligned}
& A_{1}^{ \pm} I_{0}^{-}+A_{2}^{ \pm} I_{1}^{-}+A_{3}^{ \pm} I_{2}^{-}=0, \\
& A_{1}^{ \pm} I_{0}^{+}-A_{2}^{ \pm} I_{1}^{+}+A_{3}^{ \pm} I_{2}^{+}=0,
\end{aligned}
$$

where

$$
I_{j}^{ \pm}=\int_{1}^{1 / k} \frac{\xi^{j} d \xi}{\left(\xi \pm \zeta_{\infty}\right)^{2} \sqrt{\left|p_{2}(\xi)\right|}}, \quad j=0,1,2 .
$$

The system of eight equations (3.8), (3.13) for the six unknowns $A_{j}^{ \pm}(j=1,2,3)$ has rank 6: the third and fourth equations in (3.13) are identically satisfied provided $A_{j}^{ \pm}$solve equations (3.8) and the first and second equations in (3.13). Upon solving the system we express the coefficients $A_{j}^{ \pm}$ through the four problem parameters $\alpha^{ \pm}$and $\beta^{ \pm}$and the four conformal map parameters $c^{\prime}, c^{\prime \prime}$, $\zeta_{\infty}$, and $k$ in the form

$$
\begin{gathered}
A_{1}^{ \pm}=\frac{d^{ \pm}}{\lambda_{0}}\left[\zeta_{\infty}\left(\lambda_{1} \zeta_{\infty}-2\right) I_{1}^{-}+\left(1-\lambda_{1} \zeta_{\infty}\right) I_{2}^{-}\right] \\
A_{2}^{ \pm}=\frac{d^{ \pm}}{\lambda_{0}}\left[\zeta_{\infty}\left(2-\lambda_{1} \zeta_{\infty}\right) I_{0}^{-}+\lambda_{1} I_{2}^{-}\right], \quad A_{3}^{ \pm}=-\frac{d^{ \pm}}{\lambda_{0}}\left[\left(1-\lambda_{1} \zeta_{\infty}\right) I_{0}^{-}+\lambda_{1} I_{1}^{-}\right] .
\end{gathered}
$$

Here,

$$
\lambda_{0}=\zeta_{\infty}^{2} I_{0}^{-}-2 \zeta_{\infty} I_{1}^{-}+I_{2}^{-}, \quad \lambda_{1}=\frac{p_{2}^{\prime}\left(\zeta_{\infty}\right)}{2 p_{2}\left(\zeta_{\infty}\right)} .
$$

The map itself, in addition to the four real parameters $c^{\prime}, c^{\prime \prime}, \zeta_{\infty}$, and $k$, has an additive constant, $B$,

$$
\omega(\zeta)=\frac{1}{2 \bar{a}}\left[-\frac{A_{0}}{\zeta-\zeta_{\infty}}+\int_{\zeta_{0}}^{\zeta} \frac{\left(A_{1}+A_{2} \xi+A_{3} \xi^{2}\right) d \xi}{\left(\xi-\zeta_{\infty}\right)^{2} \sqrt{p_{2}(\xi)}}\right]+B,
$$

where the path of integration $\zeta_{0} \zeta$ does not pass through the point $\zeta_{\infty} \in(-1,1)$. When a point $\zeta$ traverses the contours $l_{0}$ or $l_{1}$, the point $z=\omega(\zeta)$ traverses the contours $L_{0}$ or $L_{1}$, respectively.

The function $\psi(\zeta)$ may have inadmissible poles in the exterior of $l$. They coincide with the zeros of the derivative $\omega^{\prime}(\zeta)$ or, equivalently, with the zeros of the function

$$
\eta(\zeta)=A_{1}+A_{2} \zeta+A_{3} \zeta^{2}+A_{0} p_{2}^{1 / 2}(\zeta) .
$$

The number of inadmissible poles of the function $\psi(\zeta)$ is determined by

$$
Z=\frac{1}{2 \pi i}\left(\int_{l_{0}}+\int_{l_{1}}+\lim _{R \rightarrow \infty} \int_{\Gamma_{R}}\right) \frac{\eta^{\prime}(\zeta) d \zeta}{\eta(\zeta)},
$$

where the positive direction is chosen such that the interior of circle $\Gamma_{R}=\{|\zeta|=R\}$ and the exterior of the cuts $l$ is on the left. Noticing that

$$
\eta^{\prime}(\zeta)=A_{2}+2 A_{3} \zeta+\frac{A_{0} \zeta\left(2 \zeta^{2}-1-1 / k^{2}\right)}{p_{2}^{1 / 2}(\zeta)} \sim 2\left(A_{0}+A_{3}\right) \zeta, \quad \zeta \rightarrow \infty,
$$

we can transform formula (3.19) as

$$
Z=2+\frac{1}{2 \pi i}\left(\int_{-1 / k}^{-1}-\int_{1}^{1 / k}\right)\left(\frac{\eta_{0}^{-}(\xi)}{\eta^{-}(\xi)}-\frac{\eta_{0}^{+}(\xi)}{\eta^{+}(\xi)}\right) \frac{d \xi}{\sqrt{\left|p_{2}(\xi)\right|}} .
$$




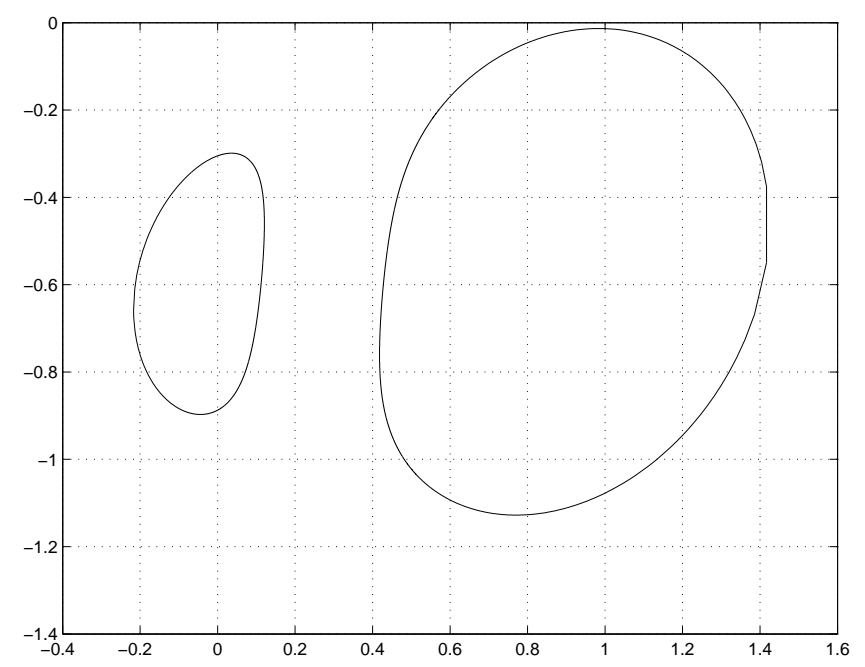

Figure 2: Two equal-strength holes when $\zeta_{\infty}=0.5, p=\tau=0, \sigma_{1}^{\infty}=\sigma_{2}^{\infty}=1, \tau^{\infty}=0.1(\gamma=0.1)$, $k=0.01, c^{\prime}=1, c^{\prime \prime}=0$.

where

$$
\begin{gathered}
\eta^{ \pm}(\xi)= \pm i \sqrt{\left|p_{2}(\xi)\right|} A_{0}+A_{1}+A_{2} \xi+A_{3} \xi^{2} \\
\eta_{0}^{ \pm}(\xi)=\mp i A_{0} \xi\left(2 \xi^{2}-1-1 / k^{2}\right)+\sqrt{\left|p_{2}(\xi)\right|}\left(A_{2}+2 A_{3} \xi\right) .
\end{gathered}
$$

On evaluating the integrals in (3.21) we conclude that if $\gamma=|b / a|<1$, then $Z=0$, and the function $\psi(\zeta)$ is analytic in the exterior of the cuts $l=l_{0} \cup l_{1}$ and continuous up to the boundary $l$. When $\gamma>1$, the function $\psi(\zeta)$ has four inadmissible poles; the solution does not exist. Finally, if $\gamma=1$, then the contours $L_{0}$ and $L_{1}$ are two straight segments, and the four poles become removable singularities of the function $\psi(\zeta)$ lying in the loops $l_{0}$ and $l_{1}$.

The profiles $L_{j}$ of equal-strength holes are determined from formula (3.17) as

$$
\begin{gathered}
z=\frac{1}{2 \bar{a}}\left[-\frac{A_{0}}{\zeta-\zeta_{\infty}}+I_{-} \pm i J(-1 / k, \zeta)\right], \quad \zeta \in l_{0}^{ \pm}, \quad z \in L_{0}, \\
z=\frac{1}{2 \bar{a}}\left[-\frac{A_{0}}{\zeta-\zeta_{\infty}}+I_{+} \mp i J(1, \zeta)\right], \quad \zeta \in l_{1}^{ \pm}, \quad z \in L_{1},
\end{gathered}
$$

where

$$
I_{ \pm}=\int_{\Gamma_{ \pm}} \frac{\left(A_{1}+A_{2} \xi+A_{3} \xi^{2}\right) d \xi}{\left(\xi-\zeta_{\infty}\right)^{2} p_{2}^{1 / 2}(\xi)}, \quad J(d, \zeta)=\int_{d}^{\zeta} \frac{\left(A_{1}+A_{2} \xi+A_{3} \xi^{2}\right) d \xi}{\left(\xi-\zeta_{\infty}\right)^{2} \sqrt{\left|p_{2}(\xi)\right|}}
$$

Here, $\Gamma_{ \pm}$are the segments with the staring and terminal points $\zeta_{0}$ and \pm 1 , respectively, and with no loss we assume $B=0$ and $\zeta_{0}=-i$.

Some typical shapes of two nonsymmetric equal-strength holes when $\zeta_{\infty}$ is a finite point are shown in Figures 2 and 3. In Figures 2 and 3 (b) and (c), the cavities are of different area and not symmetric due to the shift of the point $\zeta_{\infty}$ with respect to the center $\zeta=0$. In case 3 (a) $\zeta_{\infty}=0$, and the cavities have the same shape and area but not symmetric with respect to the real and imaginary axes due to the nonzero tangential stress $\tau^{\infty}$ applied at infinity. In the cases shown in Figures 2 and 3 (a) - (c) $\gamma<1$, and the function $\Psi(z)$ does not have poles in the exterior of the holes. Referring to Figure 3(d), we observe that the contours $L_{0}$ and $L_{1}$ intersect each other, that is in the case $\gamma>1$ the presence of inadmissible poles of the function $\Psi(z)$ may not be the only one feature which indicates that the solution does not exist. When $\gamma$ approaches 1 and either $\gamma<1$ or $\gamma>1$, the contours become slim, and in the limit, when $\gamma=1$, the contours $L_{0}$ and $L_{1}$ become segments, and the function $\Psi(z)$ is continuous everywhere in $D^{e}$ up to the boundary. 

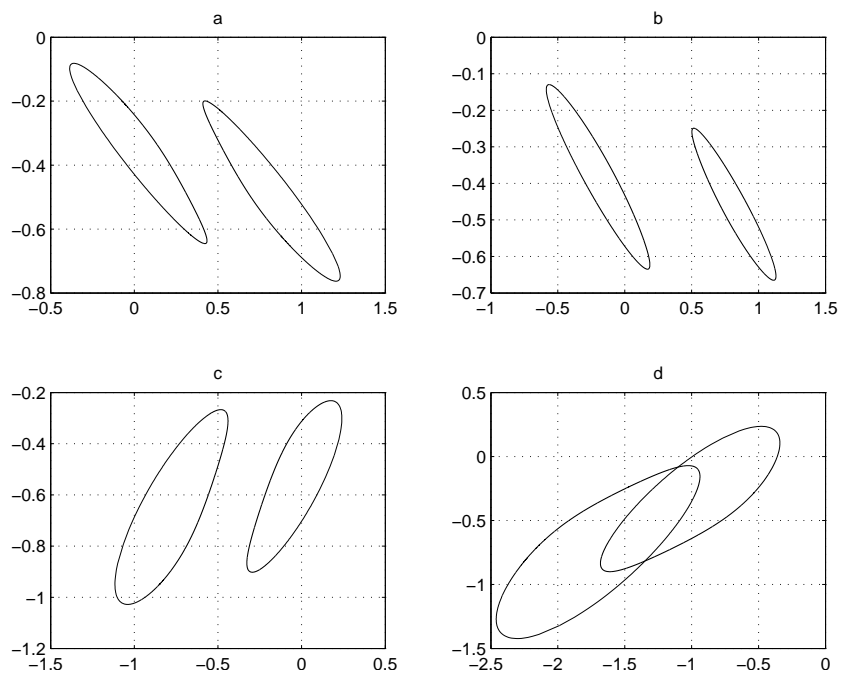

Figure 3: Two equal-strength holes when $\zeta_{\infty}$ is a finite point, $p=\tau=0, c^{\prime}=1$, and $c^{\prime \prime}=0$. (a): $\sigma_{1}^{\infty}=2, \sigma_{2}^{\infty}=1, \tau^{\infty}=-1(\gamma=0.745360), k=0.01, \zeta_{\infty}=0 ;(b): \sigma_{1}^{\infty}=2, \sigma_{2}^{\infty}=1, \tau^{\infty}=-1$ $(\gamma=0.745360), k=0.1, \zeta_{\infty}=-0.1 ;(\mathrm{c}): \sigma_{1}^{\infty}=\sigma_{2}^{\infty}=2, \tau^{\infty}=1(\gamma=0.5), k=0.01, \zeta_{\infty}=-0.1$; (d): $\sigma_{1}^{\infty}=\sigma_{2}^{\infty}=1, \tau^{\infty}=2(\gamma=2), k=0.01, \zeta_{\infty}=-0.1$. In case (d) $\gamma>1$, the function $\Psi(z)$ has four inadmissible poles, and the solution does not exist.

\subsection{Two cavities when $\zeta_{\infty}$ is a finite point: the symmetric case}

The solution may be significantly simplified in the symmetric case when $c^{\prime \prime}=0, \zeta_{\infty}=0, \tau=\tau^{\infty}=$ 0. Then

$$
\begin{gathered}
a=\frac{\sigma-p}{2}, \quad b=\frac{\sigma_{2}^{\infty}-\sigma_{1}^{\infty}}{2}, \quad \alpha^{ \pm}=b \pm a, \quad \beta^{ \pm}=0, \\
d^{+}=\frac{c^{\prime} \alpha^{+}}{k}, \quad d^{-}=0, \quad A_{0}^{+}=0, \quad A_{0}^{-}=-c^{\prime} \alpha^{-}, \\
A_{1}^{+}=\frac{c^{\prime} \alpha^{+}}{k}, \quad A_{1}^{-}=0, \quad A_{2}^{ \pm}=0, \quad A_{3}^{-}=0, \quad A_{3}^{+}=-\frac{c^{\prime} \alpha^{+} I_{0}^{-}}{k I_{2}^{-}},
\end{gathered}
$$

and the derivative of the conformal map has the form

$$
\omega^{\prime}(\zeta)=\frac{c^{\prime}}{2 a \zeta^{2}}\left[\alpha^{-}+\frac{\alpha^{+}\left(1-\zeta^{2} I_{0}^{-} / I_{2}^{-}\right)}{k p_{2}^{1 / 2}(\zeta)}\right] .
$$

Two sample symmetric holes are represented in Figure 4. For the parameters chosen, as $k \rightarrow 0$, ellipse-like holes deform into "kidney"-like cavities known in the literature [3], 17], 7].

It can be directly verified that $\sigma_{t}=\sigma, \sigma_{n}=p$, and $\tau_{n t}=\tau, z \in L_{j}, j=0,1$. Indeed, on using (2.1) to (2.5) we have

$$
\sigma_{t}=\frac{\sigma+p}{2}+\operatorname{Re} a, \quad \sigma_{n}=\sigma+p-\sigma_{t}, \quad \tau_{t n}=\operatorname{Im} a .
$$

For the lower half of the boundary of the right hole shown in Figure 4 , the variation of the stresses $\sigma_{1}, \sigma_{2}$, and $\tau_{12}$ given by

$$
\sigma_{1}=\frac{1}{2}(\sigma+p)-\operatorname{Re} \Psi(z), \quad \sigma_{2}=\frac{1}{2}(\sigma+p)+\operatorname{Re} \Psi(z), \quad \tau_{12}=\operatorname{Im} \Psi(z),
$$

with the arc length $s$ are represented in Figure 5, The point $z$ traverses the contour $L_{1}$ in the clock wise direction, while its preimage $\xi$ traverses the upper side of the loop $l_{1}$ with the starting and terminal points $\xi=1$ and $\xi=1 / k$, respectively. 


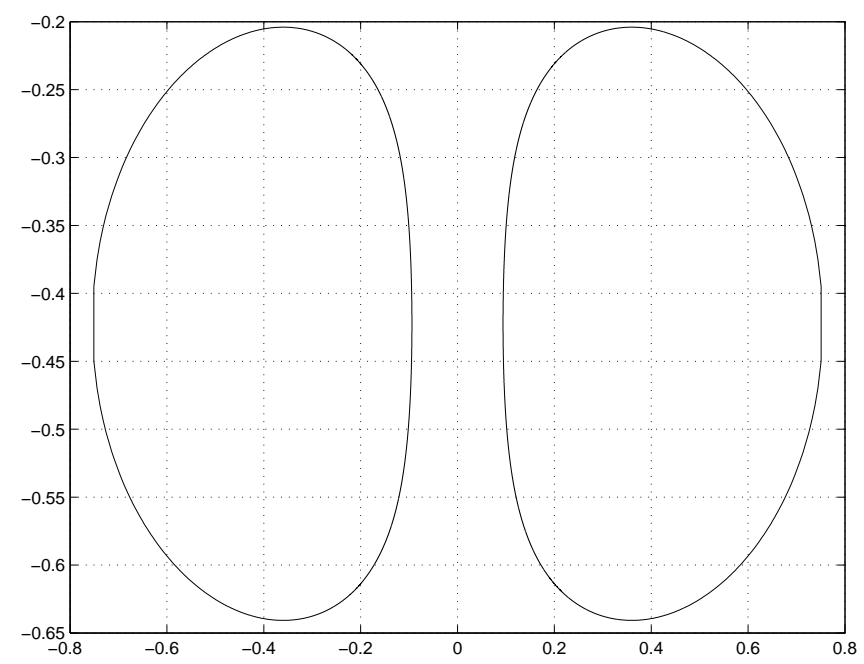

Figure 4: Two equal-strength symmetric holes for $\zeta_{\infty}=0, p=\tau=\tau^{\infty}=0, \sigma_{1}^{\infty}=2, \sigma_{2}^{\infty}=1$ $(\gamma=1 / 3), \kappa=0.01, c^{\prime}=1$, and $c^{\prime \prime}=0$.

\subsection{Two symmetric cavities when $\zeta_{\infty}=\infty$}

If it is assumed that as in [3] the function $z=\omega(\zeta)$ maps the infinite point $\zeta=\infty$ into the infinite point $z=\infty$, and the case is symmetric that is $c^{\prime \prime}=0, \tau=\tau^{\infty}=0$, then $a$ and $b$ are real,

$$
F_{-}(\zeta)=c^{\prime} \alpha^{-}, \quad F_{+}(\zeta)=\frac{c^{\prime} \alpha^{+}\left(\zeta^{2}-I_{2} / I_{0}\right)}{p^{1 / 2}(\zeta)}
$$

and the function $\omega^{\prime}(\zeta)$ can be represented as

$$
\omega^{\prime}(\zeta)=\frac{c^{\prime}}{2 a}\left[-\alpha^{-}+\frac{\alpha^{+}\left(\zeta^{2}-I_{2} / I_{0}\right)}{p_{2}^{1 / 2}(\zeta)}\right] .
$$

Here,

$$
I_{j}=\int_{1}^{1 / k} \frac{\xi^{j} d \xi}{\sqrt{\left|p_{2}(\xi)\right|}}, \quad j=0,2 .
$$

It is directly verified that the function (3.30) satisfy the conditions (3.12), and it is a one-toone map. Possible poles of the function $\psi(\zeta)$ coincide with the zeros of the derivative $\omega^{\prime}(\zeta)$ or, equivalently, with the zeros of the function

$$
\eta(\zeta)=\alpha_{1}\left(\zeta^{2}-\rho\right)-\alpha_{0} p_{2}^{1 / 2}(\zeta), \quad \alpha_{0}=\alpha^{-}, \quad \alpha_{1}=\alpha^{+}, \quad \rho=I_{2} / I_{0}
$$

Due to the symmetry of the contours $l_{0}$ and $l_{1}$ the number of inadmissible poles of the function $\psi(\zeta)$ is determined by

$$
Z=2+\frac{1}{\pi i} \int_{1}^{1 / k} \frac{\eta^{-}(\xi)\left[\eta^{\prime}(\xi)\right]^{+}-\eta^{+}(\xi)\left[\eta^{\prime}(\xi)\right]^{-}}{\eta^{+}(\xi) \eta^{-}(\xi)} d \xi
$$

where $\eta^{ \pm}(\xi)=\eta(\xi \pm i 0),\left[\eta^{\prime}(\xi)\right]^{ \pm}=\eta^{\prime}(\xi \pm i 0)$. On substituting the limiting values $\eta^{ \pm}(\xi)$ and $\left[\eta^{\prime}(\xi)\right]^{ \pm}$into the last formula we simplify it to the form $Z=2-\alpha_{0} \alpha_{1} I_{0} / \pi$, where we denoted

$$
I=2 \int_{1}^{1 / k} \frac{\left[\left(\xi^{2}-\rho\right)\left(2 \xi^{2}-1-1 / k^{2}\right)+2\left|p_{2}(\xi)\right|\right] \xi d \xi}{\left[\alpha_{1}^{2}\left(\xi^{2}-\rho\right)^{2}+\alpha_{0}^{2}\left|p_{2}(\xi)\right|\right] \sqrt{\left|p_{2}(\xi)\right|}}
$$






Figure 5: The stresses $\sigma_{1}, \sigma_{2}$, and $\sigma_{12}$ versus the arc length $s$ on the lower half of the boundary of the right hole shown in Figure 4 .

By denoting $\mu_{ \pm}=\left(1 / k^{2} \pm 1\right) / 2$ and making the substitutions first $\zeta^{2}=\mu_{-} \cos \theta+\mu_{+}$and then $w=e^{i \theta}$, we derive

$$
I=-2 i \int_{|w|=1} \frac{\left\{\left[\mu_{-}\left(w^{2}+1\right)+2 \mu_{+} w\right]\left(\mu_{+}-\rho\right)+2 w\left(\rho \mu_{+}-1 / k^{2}\right)\right\} d w}{g_{0}(w)},
$$

where

$$
g_{0}(w)=\mu_{-}^{2}\left(\alpha_{1}^{2}-\alpha_{0}^{2}\right)\left(w^{2}+1\right)^{2}+4 \alpha_{1}^{2}\left(\mu_{+}-\rho\right) \mu_{-} w\left(w^{2}+1\right)+4\left[\alpha_{1}^{2}\left(\mu_{+}-\rho\right)^{2}+\alpha_{0}^{2} \mu_{-}^{2}\right] w^{2} .
$$

This integral is evaluated by the theory of residues. The four zeros of the function $g_{0}(w)$ can be easily determined; they are

$$
w_{1,2}=\delta_{ \pm}+\sqrt{\delta_{ \pm}^{2}-1}, \quad w_{3,4}=\delta_{ \pm}-\sqrt{\delta_{ \pm}^{2}-1}
$$

and

$$
\delta_{ \pm}=\frac{\alpha_{1}^{2}\left(\rho-\mu_{+}\right) \pm \alpha_{0} \sqrt{\alpha_{1}^{2}\left(\mu_{+}-\rho\right)^{2}-\mu_{-}^{2}\left(\alpha_{1}^{2}-\alpha_{0}^{2}\right)}}{\mu_{-}\left(\alpha_{1}^{2}-\alpha_{0}^{2}\right)} .
$$

The final formula for the number of inadmissible poles of the function $\psi(\zeta)$ becomes

$$
Z=2-\alpha_{*} \sum_{j=1, \ldots, 4 ;\left|w_{j}\right|<1} \frac{\left[\mu_{-}\left(w_{j}^{2}+1\right)+2 \mu_{+} w_{j}\right]\left(\mu_{+}-\rho\right)+2 w_{j}\left(\rho \mu_{+}-1 / k^{2}\right)}{g_{j}},
$$

where $\alpha_{*}=\alpha_{0} / \alpha_{1}$ and

$$
g_{j}=\mu_{-}^{2}\left(1-\alpha_{*}^{2}\right) w_{j}\left(w_{j}^{2}+1\right)+\mu_{-}\left(\mu_{+}-\rho\right)\left(3 w_{j}^{2}+1\right)+2 w_{j}\left[\left(\mu_{+}-\rho\right)^{2}+\alpha_{*}^{2} \mu_{-}^{2}\right] .
$$

It turns out that two and only two zeros out of the four zeros $w_{j}(j=1,2,3,4)$ lie inside the unit disc $|w|<1$. As in the case when $\zeta_{\infty}$ is a finite point in the segment $(-1,1)$ if $\gamma<1$, then $Z=0$, and the function $\Psi(z)$ is analytic everywhere in the domain $D^{e}$. If $\gamma>1$, then $Z=4$, and the function $\Psi(z)$ has four simple poles in the domain $D^{e}$. In the limiting case $\gamma=1$, the function $\Psi(z)$ has removable singularities in the boundary of the domain $D^{e}$, and the contours $L_{0}$ and $L_{1}$ are straight segments.

Sample contours of symmetric equal-strength holes when $\zeta_{\infty}=\infty$ and when $\gamma<1$ are given in Figures 6 (a) - (c). In Figure 6 (d), the parameter $\gamma>1$, the function $\Psi(z)$ has four inadmissible poles, and in addition, the contours intersect each other; the solution does not exist. 

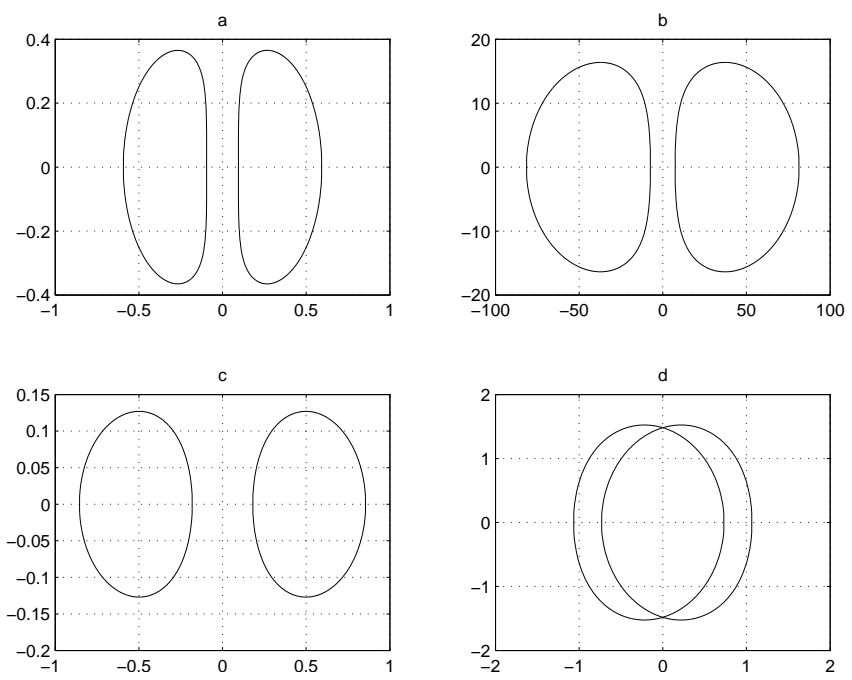

Figure 6: Two equal-strength symmetric holes for $\zeta_{\infty}=\infty, c^{\prime}=1, c^{\prime \prime}=0, \tau^{\infty}=0, a=1$. (a): $k=0.001, b=0,(\gamma=0) ;(\mathrm{b}) k=0.01, b=-0.5(\gamma=0.5) ;(\mathrm{c}) k=0.1, b=-0.5(\gamma=0.5) ;(\mathrm{d})$ $k=0.1, b=5(\gamma=5)$. In case (d) the function $\Psi(z)$ has four inadmissible poles.

\section{Three cavities: $\zeta_{\infty}$ is a finite point}

Any triply connected domain $D^{e}$ can be considered as the image by a conformal map $z=\omega(\zeta)$ of a parametric $\zeta$-plane cut along three segments in the real axis, $l_{0}=[-1 / k,-1], l_{1}=\left[k_{1}, k_{2}\right]$, and $l_{2}=[1,1 / k]$, where $0<k<1,-1<k_{1}<k_{2}<1$. Given the domain $D^{e}$ such a map is unique. The point $z=\infty$ is the image of a certain point $\zeta_{\infty}=\zeta_{\infty}^{\prime}+i \zeta_{\infty}^{\prime \prime}$, and, in general, the parameters $\zeta_{\infty}^{\prime}, \zeta_{\infty}^{\prime \prime}, k, k_{1}$, and $k_{2}$ cannot be prescribed and should be recovered from the solution.

Let $\mathcal{R}$ be the hyperelliptic surface of the algebraic function $u^{2}=p_{3}(\zeta)$, where

$$
p_{3}(\zeta)=\left(\zeta^{2}-1\right)\left(\zeta^{2}-1 / k^{2}\right)\left(\zeta-k_{1}\right)\left(\zeta-k_{2}\right) .
$$

We fix the single branch $f(\zeta)$ of the function $p_{3}^{1 / 2}(\zeta)$ in $\mathbb{C} \backslash l, l=l_{0} \cup l_{1} \cup l_{2}$, by the condition $f(\zeta) \sim \zeta^{3}, \zeta \rightarrow \infty$. The branch is pure imaginary on the cut sides,

$$
\begin{gathered}
f(\zeta)= \pm(-1)^{m} i\left|p_{3}^{1 / 2}(\xi)\right|, \quad \zeta=\xi \pm i 0, \quad \xi \in l_{m}, \quad m=0,1,2, \\
f(\xi)=\left|p_{3}^{1 / 2}(\xi)\right|, \quad-1<\xi<k_{1}, \quad f(\xi)=-\left|p_{3}^{1 / 2}(\xi)\right|, \quad k_{2}<\xi<1 .
\end{gathered}
$$

Similarly to the case $n=2$, on the surface $\mathcal{R}$, we introduce the functions (3.1) which satisfy the Riemann-Hilbert problems (3.3), (3.2). Their solution in the first sheet has the form

$$
F_{+}(\zeta)=\frac{R_{+}(\zeta)}{f(\zeta)}, \quad F_{-}(\zeta)=\frac{i R_{-}(\zeta)}{f(\zeta)}
$$

where

$$
\begin{gathered}
R_{ \pm}(\zeta)=A_{1}^{ \pm}+A_{2}^{ \pm} \zeta+A_{3}^{ \pm} \frac{f(\zeta)+f\left(\zeta_{\infty}\right)}{\zeta-\zeta_{\infty}}-A_{3}^{ \pm} \frac{f(\zeta)-f\left(\bar{\zeta}_{\infty}\right)}{\zeta-\bar{\zeta}_{\infty}}+\left(A_{4}^{ \pm}+i A_{5}^{ \pm}\right) \\
\times \frac{f(\zeta)+f\left(\zeta_{\infty}\right)+f^{\prime}\left(\zeta_{\infty}\right)\left(\zeta-\zeta_{\infty}\right)}{\left(\zeta-\zeta_{\infty}\right)^{2}}-\left(A_{4}^{ \pm}-i A_{5}^{ \pm}\right) \frac{f(\zeta)-f\left(\bar{\zeta}_{\infty}\right)-f^{\prime}\left(\bar{\zeta}_{\infty}\right)\left(\zeta-\bar{\zeta}_{\infty}\right)}{\left(\zeta-\bar{\zeta}_{\infty}\right)^{2}}
\end{gathered}
$$


where $A_{j}^{ \pm}(j=1,2, \ldots, 5)$ are free real constants. Analysis of these functions in a neighborhood of the point $\zeta_{\infty}$ yields

$$
F_{ \pm}(\zeta)=i^{1 / 2 \mp 1 / 2}\left(\frac{2\left(A_{4}^{ \pm}+i A_{5}^{ \pm}\right)}{\left(\zeta-\zeta_{\infty}\right)^{2}}+\frac{2 A_{3}^{ \pm}}{\zeta-\zeta_{\infty}}\right)+O(1), \quad \zeta \rightarrow \zeta_{\infty}
$$

By virtue of the behavior of the functions $F_{ \pm}(\zeta)=-c_{-1}(b \pm \bar{a})\left(\zeta-\zeta_{\infty}\right)^{-2}+O(1), \zeta \rightarrow \zeta_{\infty}$, required, we immediately find

$$
\begin{gathered}
A_{3}^{ \pm}=0, \quad A_{4}^{+}=-\frac{1}{2}\left(c^{\prime} \alpha^{+}-c^{\prime \prime} \beta^{+}\right), \quad A_{4}^{-}=-\frac{1}{2}\left(c^{\prime} \beta^{-}+c^{\prime \prime} \alpha^{-}\right), \\
A_{5}^{+}=-\frac{1}{2}\left(c^{\prime} \beta^{+}+c^{\prime \prime} \alpha^{+}\right), \quad A_{5}^{-}=\frac{1}{2}\left(c^{\prime} \alpha^{-}-c^{\prime \prime} \beta^{-}\right) .
\end{gathered}
$$

The coefficients $A_{1}^{ \pm}$and $A_{2}^{ \pm}$are still not determined in the expression of the function $\omega^{\prime}(\zeta)$ that is

$$
\begin{gathered}
\omega^{\prime}(\zeta)=\frac{1}{2 \bar{a} f(\zeta)}\left[A_{1}+A_{2} \zeta+\left(A_{4}+i A_{5}\right) \frac{f(\zeta)+f\left(\zeta_{\infty}\right)+f^{\prime}\left(\zeta_{\infty}\right)\left(\zeta-\zeta_{\infty}\right)}{\left(\zeta-\zeta_{\infty}\right)^{2}}\right. \\
\left.-\left(A_{4}-i A_{5}\right) \frac{f(\zeta)-f\left(\bar{\zeta}_{\infty}\right)-f^{\prime}\left(\bar{\zeta}_{\infty}\right)\left(\zeta-\bar{\zeta}_{\infty}\right)}{\left(\zeta-\bar{\zeta}_{\infty}\right)^{2}}\right]
\end{gathered}
$$

where $A_{j}=A_{j}^{+}-i A_{j}^{-}$. To determine the coefficients $A_{1}$ and $A_{2}$, we need to guarantee that the function $z=\omega(\zeta)$ is a single-valued map that is to force the function $\omega^{\prime}(\zeta)$ to meet the three conditions

$$
\int_{l_{m}} \omega^{\prime}(\zeta) d \zeta=0, \quad m=0,1,2
$$

or, equivalently,

$$
I_{m 0} A_{1}+I_{m 1} A_{2}=-J_{m}, \quad m=0,1,2
$$

where

$$
\begin{gathered}
I_{m j}=\int_{l_{m}} \frac{\xi^{j} d \xi}{f(\xi)}, \quad j=0,1 \\
J_{m}=\left(A_{4}+i A_{5}\right) \int_{l_{m}} \frac{\left[f\left(\zeta_{\infty}\right)+f^{\prime}\left(\zeta_{\infty}\right)\left(\xi-\zeta_{\infty}\right)\right] d \xi}{f(\xi)\left(\xi-\zeta_{\infty}\right)^{2}} \\
+\left(A_{4}-i A_{5}\right) \int_{l_{m}} \frac{\left[f\left(\bar{\zeta}_{\infty}\right)+f^{\prime}\left(\bar{\zeta}_{\infty}\right)\left(\xi-\bar{\zeta}_{\infty}\right)\right] d \xi}{f(\xi)\left(\xi-\bar{\zeta}_{\infty}\right)^{2}}, \quad m=0,1,2 .
\end{gathered}
$$

The first two equations in (4.9) constitute an inhomogeneous system of two complex equations with respect to complex constants $A_{1}$ and $A_{2}$. The coefficients of the system, the integrals $I_{m j}$ $(j, m=0,1)$ are the $A$-periods of the abelian integrals

$$
\int_{(1 / k, 0)}^{(\zeta, u(\zeta))} \frac{\xi^{j} d \xi}{u(\xi)}, \quad j=0,1
$$

associated with the genus-2 Riemann surface $\mathcal{R}$ of the algebraic function $u^{2}(\xi)=p_{3}(\xi)$. Therefore the $2 \times 2$ matrix $\left\{I_{m j}\right\}(j, m=0,1)$ is not singular, and the unique solution is given by

$$
A_{1}=\frac{J_{1} I_{01}-J_{0} I_{11}}{\Delta}, \quad A_{2}=\frac{J_{0} I_{10}-J_{1} I_{00}}{\Delta}
$$

where $\Delta=I_{00} I_{11}-I_{01} I_{10}$. The third equation in (4.9) is transformed to the form

$$
J_{1}\left(I_{01} I_{20}-I_{00} I_{21}\right)+J_{0}\left(I_{10} I_{21}-I_{11} I_{20}\right)+J_{2} \Delta=0
$$


and satisfied identically. This is due to the fact that the corresponding abelian integrals in the right hand-side in (4.9) can be represented as a linear combination of the two basis integrals (4.11).

Since the parameters $k, k_{1}, k_{2}$, and $\zeta_{\infty}=\zeta_{\infty}^{\prime}+i \zeta_{\infty}^{\prime \prime}$ are free, the derivative $\omega^{\prime}(\zeta)$ generates a five-parametric family of conformal mappings (we do not count the two free scaling parameters $\left.c_{-1}=c^{\prime}+i c^{\prime \prime}\right)$ which transforms the slit domain $\mathbb{C} \backslash l$ into the triple connected domain $D^{e}$. By integrating the function (4.7) we find the integral representation of the conformal map

$$
\begin{gathered}
\omega(\zeta)=\frac{1}{2 \bar{a}}\left\{-\frac{A_{4}+i A_{5}}{\zeta-\zeta_{\infty}}+\frac{A_{4}-i A_{5}}{\zeta-\bar{\zeta}_{\infty}}+\int_{\zeta_{0}}^{\zeta}\left[A_{1}+A_{2} \xi+\left(A_{4}+i A_{5}\right)\right.\right. \\
\left.\left.\times \frac{f\left(\zeta_{\infty}\right)+f^{\prime}\left(\zeta_{\infty}\right)\left(\xi-\zeta_{\infty}\right)}{\left(\xi-\zeta_{\infty}\right)^{2}}+\left(A_{4}-i A_{5}\right) \frac{f\left(\bar{\zeta}_{\infty}\right)+f^{\prime}\left(\bar{\zeta}_{\infty}\right)\left(\xi-\bar{\zeta}_{\infty}\right)}{\left(\xi-\bar{\zeta}_{\infty}\right)^{2}}\right] \frac{d \xi}{f(\xi)}\right\} .
\end{gathered}
$$

To find the actual profile of the holes $L_{m}$, we let $\zeta$ run the cuts $l_{m}$ and obtain

$$
\begin{gathered}
z=\mathcal{I}(\zeta) \pm i \mathcal{J}(-1 / k, \zeta), \quad \zeta \in l_{0}^{\mp}, \quad z \in L_{0}, \\
z=\mathcal{I}(\zeta)+\mathcal{J}\left(-1, k_{1}\right) \mp i \mathcal{J}\left(k_{1}, \zeta\right), \quad \zeta \in l_{1}^{\mp}, \quad z \in L_{1}, \\
z=\mathcal{I}(\zeta)+\mathcal{J}\left(-1, k_{1}\right)-\mathcal{J}\left(k_{2}, 1\right) \pm i \mathcal{J}(1, \zeta), \quad \zeta \in l_{2}^{\mp}, \quad z \in L_{2},
\end{gathered}
$$

where we denoted

$$
\begin{gathered}
\mathcal{I}(\zeta)=\frac{1}{2 \bar{a}}\left(-\frac{A_{4}+i A_{5}}{\zeta-\zeta_{\infty}}+\frac{A_{4}-i A_{5}}{\zeta-\bar{\zeta}_{\infty}}\right) \\
\mathcal{J}(d, \zeta)=\frac{1}{2 \bar{a}} \int_{d}^{\zeta}\left[A_{1}+A_{2} \xi+\left(A_{4}+i A_{5}\right) \frac{f\left(\zeta_{\infty}\right)+f^{\prime}\left(\zeta_{\infty}\right)\left(\xi-\zeta_{\infty}\right)}{\left(\xi-\zeta_{\infty}\right)^{2}}\right. \\
\left.+\left(A_{4}-i A_{5}\right) \frac{f\left(\bar{\zeta}_{\infty}\right)+f^{\prime}\left(\bar{\zeta}_{\infty}\right)\left(\xi-\bar{\zeta}_{\infty}\right)}{\left(\xi-\bar{\zeta}_{\infty}\right)^{2}}\right] \frac{d \xi}{|f(\xi)|}
\end{gathered}
$$

The function $\omega^{\prime}(\zeta)$ may not have zeros in the slit domain $\mathbb{C} \backslash l$. Otherwise the functions $\psi(\zeta)$ has unacceptable poles. As in the case of doubly connected domain we introduce a function $\eta(\zeta)$ which share the zeros with the function $\omega^{\prime}(\zeta)$ and is free of singularities of $\omega^{\prime}(\zeta)$,

$$
\omega^{\prime}(\zeta)=\frac{\eta(\zeta)}{2 \bar{a} f(\zeta)\left(\zeta-\zeta_{\infty}\right)^{2}\left(\zeta-\bar{\zeta}_{\infty}\right)^{2}}
$$

where

$$
\begin{aligned}
& \eta(\zeta)=\left(A_{1}+A_{2} \zeta\right)\left(\zeta-\zeta_{\infty}\right)^{2}\left(\zeta-\bar{\zeta}_{\infty}\right)^{2}+\left(A_{4}+i A_{5}\right)\left(\zeta-\bar{\zeta}_{\infty}\right)^{2}\left[f(\zeta)+f\left(\zeta_{\infty}\right)\right. \\
& \left.+f^{\prime}\left(\zeta_{\infty}\right)\left(\zeta-\zeta_{\infty}\right)\right]-\left(A_{4}-i A_{5}\right)\left(\zeta-\zeta_{\infty}\right)^{2}\left[f(\zeta)-f\left(\bar{\zeta}_{\infty}\right)-f^{\prime}\left(\bar{\zeta}_{\infty}\right)\left(\zeta-\bar{\zeta}_{\infty}\right)\right] .
\end{aligned}
$$

The zero counting formula applied yields that the number of zeros, $Z$, of the function $\eta(\zeta)$ in the slit domain is

$$
Z=5+\frac{1}{2 \pi i} \sum_{m=0}^{2} \int_{l_{m}} \frac{\eta^{\prime}(\zeta) d \zeta}{\eta(\zeta)} .
$$

Here, we used the asymptotics at infinity

$$
\begin{gathered}
f(\zeta) \sim \zeta^{3}, \quad f^{\prime}(\zeta) \sim 3 \zeta^{2}, \\
\eta(\zeta) \sim\left(A_{2}+2 i A_{5}\right) \zeta^{5}, \quad \eta^{\prime}(\zeta) \sim 5\left(A_{2}+2 i A_{5}\right) \zeta^{4}, \quad \zeta \rightarrow \infty
\end{gathered}
$$

and the limit as $R \rightarrow \infty$ of the integral over a circle $\Gamma_{R}$ of radius $R$ centered at the origin

$$
\lim _{R \rightarrow \infty} \int_{R} \frac{\eta^{\prime}(\zeta) d \zeta}{\eta(\zeta)}=5 .
$$



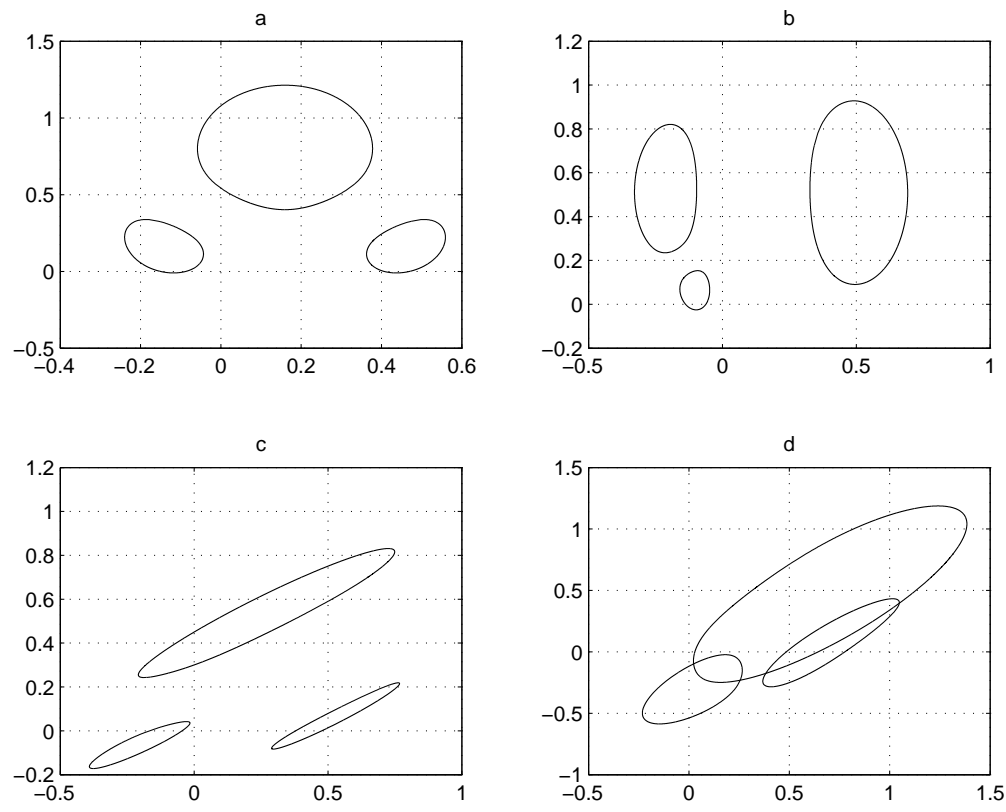

Figure 7: The contours $L_{0}, L_{1}$, and $L_{2}$ when $\zeta_{\infty}$ is a finite point, $c^{\prime}=1, c^{\prime \prime}=0, k_{1}=-0.8$, $k=0.2, k_{2}=0.8, p=\tau=0 .(\mathrm{a}): \zeta_{\infty}=i, \sigma_{1}^{\infty}=1, \sigma_{2}^{\infty}=2, \tau^{\infty}=0(\gamma=1 / 3) ;(\mathrm{b}): \zeta_{\infty}=1+i$, $\sigma_{1}^{\infty}=1, \sigma_{2}^{\infty}=2, \tau^{\infty}=0(\gamma=1 / 3) ;(\mathrm{c}): \zeta_{\infty}=i, \sigma_{1}^{\infty}=2, \sigma_{2}^{\infty}=1, \tau^{\infty}=1(\gamma=0.7453560) ;(\mathrm{d})$ : $\zeta_{\infty}=i, \sigma_{1}^{\infty}=\sigma_{2}^{\infty}=1, \tau^{\infty}=2(\gamma=2)$. In cases (a) - (c) the function $\Psi(z)$ has two inadmissible poles in the domain $D^{e}$, while in case (d) the number of poles is 8 .

On computing the integrals in (4.19) it is possible to establish that if $\gamma=|b / a|<1$, then $Z=2$, and when $\gamma>1$, the function $\omega^{\prime}(\zeta)$ has even more zeros, $Z=8$. In the limiting case $\gamma=1$, these points lying in the contour $l$ are removable singularities of the function $\psi(\zeta)$ due to the relation $\overline{F_{+}(\xi)+F_{-}(\xi)}=-\left[F_{+}(\xi)-F_{-}(\xi)\right], \xi \in l$. When $\gamma=1$, the contours $L_{j}$ are straight segments in the $z$-plane.

Thus, the conformal map which transforms any finite point $\zeta_{\infty}$ of the slit domain into the infinite point $z=\infty$ gives rise to a finite number of poles of the function $\Psi(z)$ regardless of the value of the parameter $\gamma \neq 1$. This means that such a family of maps cannot be employed to identify equal-strength cavities. At the same time, when $\gamma<1$, the map $z=f(\zeta)$ given by (4.15) generates some contours $L_{m}$ which do not intersect each other. Samples of such contours when $\gamma<1$ and the function $\Psi(z)$ has two inadmissible poles in the domain $D^{e}$ are given in Figure 7 (a) - (c). Shown in Figure 7 (d) the three loops intersect each other, $\gamma>1$, and the function $\Psi(z)$ has eight inadmissible poles in the domain $D^{e}$.

\section{$5 \quad n$ cavities: domain $D_{0}^{e}, n \geq 3$, and $\zeta_{\infty}=\infty$}

The family of mappings derived in the previous section gives rise to unacceptable poles of the complex potential $\Psi(z)$. All the mappings share the same property, the infinite point $z=\infty$ is the image of a certain finite point $\zeta_{\infty}$ in the slit domain. Since the case $\omega(\infty)=\infty$ cannot be extracted from the solution derived in Section 4, we consider this case separately. Also, for generality, we assume that $n$ is not just equal to 3 , but any finite integer $n \geq 3$. We confine ourselves to the family of domains, $D_{0}^{e} \subset D^{e}$, which are the images of slit domains $\mathcal{D}^{e}$ such that all the $n$ slits lie in the same line, $\mathcal{D}^{e}=\mathbb{C} \backslash l, l=l_{0} \cup \ldots l_{n-1}$, and $l_{j}=\left[k_{2 j}, k_{2 j+1}\right], j=0, \ldots, n-1, k_{2 n-1}=-k_{0}=1$, $-1<k_{1}<\ldots<k_{2 n-2}<1$. The function $\omega(\zeta)$ has a simple pole in the vicinity of the infinite 
point and for large $z$ can be represented by (2.7). We emphasize that not every triply connected domain $D^{e}$ is the image of a slit domain $\mathcal{D}^{e}$ such that $z=\infty$ is the image of $\zeta=\infty$. Needless to say that not every $n$-connected $(n \geq 4)$ domain $D^{e}$ is the image of the exterior of $n$ slits lying in the same line. On studying the family of mappings $\omega: \mathcal{D}^{e} \rightarrow D_{0}^{e}$ when $\omega(\infty)=\infty$ we try to find a set of equal-strength holes such that $\omega^{\prime}(\zeta)$ does not have zeros in $\mathcal{D}^{e}$, and therefore the function $\Psi(z)$ is analytic everywhere in the domain $D_{0}^{e}$.

First we fix the branch $f(\zeta)$ of the function $p_{n}^{1 / 2}(\zeta)$,

$$
p_{n}(\zeta)=\prod_{j=0}^{2 n-1}\left(\zeta-k_{j}\right)
$$

in the domain $\mathcal{D}^{e}$ by the condition $f(\zeta) \sim \zeta^{n}$. The functions $F_{ \pm}(\zeta)$ are bounded at infinity, and their counterparts defined in the Riemann surface have simple poles at the branch points of the surface $\mathcal{R}$. We have

$$
F_{+}(\zeta)=\frac{1}{f(\zeta)} \sum_{j=1}^{n+1} A_{j}^{+} \zeta^{j-1}+i A_{0}^{+}, \quad F_{-}(\zeta)=\frac{i}{f(\zeta)} \sum_{j=1}^{n+1} A_{j}^{-} \zeta^{j-1}+A_{0}^{-},
$$

where $A_{j}^{ \pm}$are arbitrary real constants. By expanding these functions for large $z$ and comparing these expansions with the asymptotics of $F_{ \pm}(\zeta)$ in $(2.12)$ we find

$$
\begin{gathered}
A_{n}^{ \pm}=-k A_{n+1}^{ \pm}, \quad A_{n+1}^{-}=\alpha^{-} c^{\prime \prime}+\beta^{-} c^{\prime}, \quad A_{n+1}^{+}=\alpha^{+} c^{\prime}-\beta^{+} c^{\prime \prime}, \\
A_{0}^{-}=\alpha^{-} c^{\prime}-\beta^{-} c^{\prime \prime}, \quad A_{0}^{+}=\alpha^{+} c^{\prime \prime}+\beta^{+} c^{\prime},
\end{gathered}
$$

where $k=\frac{1}{2}\left(k_{1}+k_{2}+\ldots+k_{2 n-2}\right)$. The derivative of the conformal map $\omega^{\prime}(\zeta)$

$$
\omega^{\prime}(\zeta)=\frac{1}{2 \bar{a}}\left[i A_{0}^{+}-A_{0}^{-}+\frac{1}{f(\zeta)} \sum_{j=1}^{n+1}\left(A_{j}^{+}-i A_{j}^{-}\right) \zeta^{j-1}\right]
$$

has to generate a one-to-one map. This is guaranteed by the following $n$ complex conditions:

$$
\int_{l_{m}} \omega^{\prime}(\zeta) d \zeta=0, \quad m=0,1, \ldots, n-1
$$

These conditions can be rewritten as

$$
\sum_{j=0}^{n} a_{m j}\left(A_{j+1}^{+}-i A_{j+1}^{-}\right)=0, \quad m=0,1, \ldots, n-1 .
$$

Here,

$$
a_{m j}=\int_{l_{m}} \frac{\zeta^{j} d \zeta}{f(\zeta)}, \quad m=0,1, \ldots, n-1, \quad j=0,1, \ldots, n .
$$

The integrals $a_{m j}(m, j=0,1, \ldots, n-2)$ are the $A$-periods of the abelian integrals

$$
\int_{(1,0)}^{(\zeta, u(\zeta))} \frac{\xi^{j} d \xi}{u(\xi)}, \quad j=0,1, \ldots, n-2,
$$

associated with the genus- $(n-1)$ Riemann surface $\mathcal{R}$ of the algebraic function $u^{2}(\xi)=p_{n}(\xi)$. Therefore the matrix $a_{m j}(m, j=0,1, \ldots, n-2)$ is not singular. Denote

$$
I_{m j}=\int_{l_{m}^{+}} \frac{\xi^{j} d \xi}{|f(\xi)|}, \quad m=0,1, \ldots, n-1, \quad j=0,1, \ldots, n .
$$


The coefficients $A_{j}^{ \pm}$are uniquely determined through the known coefficients $A_{n+1}^{ \pm}$from the nonsingular system

$$
\sum_{j=0}^{n-2} I_{m j} A_{j+1}^{ \pm}=-\left(I_{m n}-k I_{m n-1}\right) A_{n+1}^{ \pm}, \quad m=0,1, \ldots, n-2 .
$$

The last equation in (5.6) is satisfied automatically because the basis of the abelian integrals (5.8) has dimension $n-1$, and the $n \times n$ matrix

$$
\left(\begin{array}{cccc}
I_{00} & \cdots & I_{0 n-2} & I_{0 n}-k I_{0 n-1} \\
\ldots & \cdots & \cdots & \cdots \\
I_{n-10} & \cdots & I_{n-1 n-2} & I_{n-1 n}-k I_{n-1 n-1}
\end{array}\right)
$$

is singular.

To determine the number of zeros of the function $\omega^{\prime}(\zeta)$, we introduce the function

$$
\eta(\zeta)=\sum_{j=1}^{n+1}\left(A_{j}^{+}-i A_{j}^{-}\right) \zeta^{j-1}+\left(i A_{0}^{+}-A_{0}^{-}\right) f(\zeta) .
$$

The functions $\eta(\zeta)$ and $\omega^{\prime}(\zeta)$ share their zeros. The number of zeros of the function $\eta(\zeta)$ coincides with the number of inadmissible poles of the function $\psi(\zeta)$ and is given by

$$
Z=\frac{1}{2 \pi i}\left(\sum_{j=0}^{n-1} \int_{l_{j}}+\lim _{R \rightarrow \infty} \int_{\Gamma_{R}}\right) \frac{\eta^{\prime}(\zeta) d \zeta}{\eta(\zeta)}=n+\frac{1}{2 \pi i} \sum_{j=0}^{n-1} \int_{l_{j}} \frac{\eta^{\prime}(\zeta) d \zeta}{\eta(\zeta)} .
$$

Our numerical tests implemented for the case $n=3$ reveal that if $\gamma=|b / a|<1$, then $Z=0$, and the function $\Psi(z)$ is analytic in $D_{0}^{e}$ and continuous up to the boundary. If $\gamma>1$, then $Z=6$, and the function $\Psi(z)$ has six poles in the domain $D_{0}^{e}$. When $|a|=|b|$, the domain $D_{0}^{e}$ is a set of straight segments, and the function $\Psi(z)$ has removable singularities in the boundary of $D_{0}^{e}$. As in the simply and doubly connected cases, if $|a|<|b|$, then the problem does not have solutions. When $|a|>|b|$, the solution exists, and the conformal map $z=\omega(\zeta)$ is defined up to seven arbitrary constants, an additive constant, two scaling parameters $c^{\prime}$ and $c^{\prime \prime}$, and the four parameters $k_{j}(j=1, \ldots, 4)$. Integrating (5.4) and employing (5.5), we have

$$
\begin{gathered}
z=\frac{1}{2 \bar{a}}\left[\left(i A_{0}^{+}-A_{0}^{-}\right) \zeta \mp i J(-1, \zeta)\right]+B, \quad \zeta \in l_{0}^{ \pm}, \quad z \in L_{0}, \\
z=\frac{1}{2 \bar{a}}\left[\left(i A_{0}^{+}-A_{0}^{-}\right) \zeta+J\left(k_{1}, k_{2}\right) \pm i J\left(k_{2}, \zeta\right)\right]+B, \quad \zeta \in l_{1}^{ \pm}, \quad z \in L_{1}, \\
z=\frac{1}{2 \bar{a}}\left[\left(i A_{0}^{+}-A_{0}^{-}\right) \zeta+J\left(k_{1}, k_{2}\right)-J\left(k_{3}, k_{4}\right) \mp i J\left(k_{4}, \zeta\right)\right]+B, \quad \zeta \in l_{2}^{ \pm}, \quad z \in L_{2} .
\end{gathered}
$$

Here, $B$ is an additive constant and without loss can be taken zero, and $J$ is the real integral

$$
J(\alpha, \beta)=\int_{\alpha}^{\beta} \frac{1}{|f(\xi)|} \sum_{j=1}^{4}\left(A_{j}^{+}-i A_{j}^{-}\right) \xi^{j-1} d \xi .
$$

Figures 8 (a)-(c) show how the change of the loading parameter $\gamma$ and the conformal mapping parameters affects the profiles of equal-strength cavities in the case $n=3$ and when $\zeta_{\infty}=\infty$. Figure 8 (d) gives a sample of the contours $L_{j}$ when $\gamma>1$. Although the contours $L_{j}$ do not have common points, the function $\Psi(z)$ has six poles in the domain $D^{e}$, and the solution does not exist. 

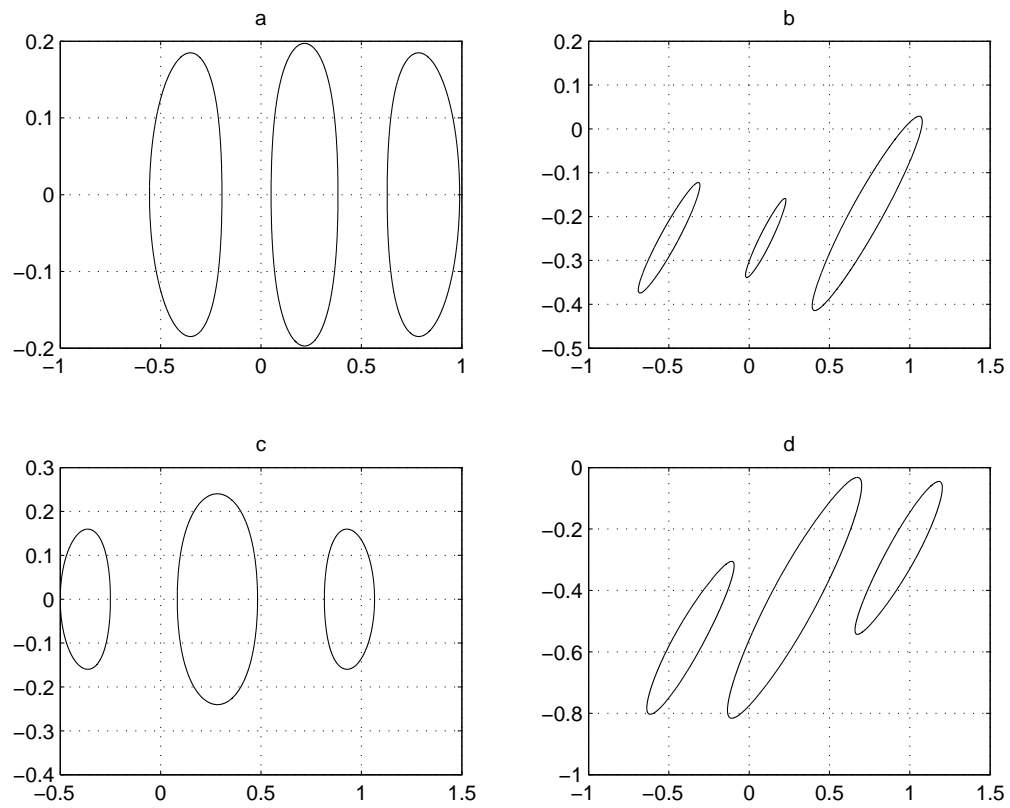

Figure 8: Three equal-strength holes when $\zeta_{\infty}=\infty, c^{\prime}=1$, and $c^{\prime \prime}=0, \tau=0$. (a): $\sigma_{1}^{\infty}=0$, $\sigma_{2}^{\infty}=1, \tau^{\infty}=0, p=5(\gamma=1 / 9), k_{1}=-k_{4}=-0.35, k_{2}=-k_{3}=-0.3 ;(\mathrm{b}): \sigma_{1}^{\infty}=2, \sigma_{2}^{\infty}=1$, $\tau^{\infty}=1, p=0(\gamma=0.745360), k_{1}=-0.5, k_{2}=-0.3, k_{3}=0, k_{4}=0.1 ;(\mathrm{c}): \sigma_{1}^{\infty}=\sigma_{2}^{\infty}=1, \tau^{\infty}=0$, $p=5(\gamma=0), k_{1}=-k_{4}=-0.5, k_{2}=-k_{3}=-0.4 ;(\mathrm{d}): \sigma_{1}^{\infty}=\sigma_{2}^{\infty}=1, \tau^{\infty}=1.5, p=0(\gamma=1.5)$, $k_{1}=-k_{4}=-0.5, k_{2}=-k_{3}=-0.4$. In cases (a) - (c) the function $\Psi(z)$ is analytic, while in case (d) the function $\Psi(z)$ has six inadmissible poles.

Conclusions. We have analyzed the inverse plane problem of constructing $n$ equal-strength cavities in an unbounded elastic body when constant loading is applied at infinity and to the cavities boundary. By advancing the method of conformal mappings employed in [3] for $n=1$ and $n=2$ (two symmetric holes) to general doubly and triply connected domains we have found by quadratures a four- and seven-parametric family of mappings and therefore a four- and sevenparametric family of two and three equal-strength cavities, respectively. In both cases two out of four and seven free parameters, respectively, are scaling parameters. For the doubly connected problem, the map $\omega$ transforms a slit domain $\mathcal{D}^{e}$, the exterior of two slits $[-1 / k,-1]$ and $[1,1 / k]$ $(0<k<1)$, into the elastic domain $D^{e}$, the exterior of the holes, and $\omega\left(\zeta_{\infty}\right)=\infty, \zeta_{\infty} \in(-1,1)$. For the triply connected problem, we analyzed two cases of the preimage of the infinite point, $\zeta_{\infty}$ is a finite point and $\zeta_{\infty}=\infty$. In the former case, $\mathcal{D}^{e}$ is the exterior of three slits $[-1 / k,-1],\left[k_{1}, k_{2}\right]$, and $[1,1 / k]$, while in the second case the slits are $\left[-1, k_{1}\right],\left[k_{2}, k_{3}\right]$, and $\left[k_{4}, 1\right]$. The conformal mappings are derived in terms of elliptic integrals for $n=2$ and hyperelliptic integrals for $n=3$. We have also analyzed the zeros of the derivative $\omega^{\prime}(\zeta)$ of the conformal mapping and shown that these zeros, if exist, generate inadmissible poles of the solution. If $\gamma=|b / a|<1$ ( $a$ and $b$ are complex loading parameters), then the Kolosov-Muskhelishvili potential $\Psi(z)$ is free of poles when $n=1$ and $n=2$. In the triply connected case, if the conformal map is chosen such that $\omega(\infty)=\infty$, then the centers of the cavities are located in the same line, and the condition $\gamma<1$ is necessary and sufficient for the solution to exist. If $\gamma>1$, then the function $\Psi(\zeta)$ has two, four, and six inadmissible poles in the cases $n=1, n=2$ and $n=3(\omega(\infty)=\infty)$, respectively. If $\gamma=1$, then the equal-strength cavities are straight segments, and the potential $\Psi(z)$ has removable singularities in the boundary. It has also been discovered that when $\gamma>1$ and big enough, then the contours intersect each other. If $n=3$ and $\zeta_{\infty}$ is a finite point, then $\Psi(\zeta)$ has two and eight poles for 
the cases $\gamma<1$ and $\gamma>1$, respectively, that is there does not exist a set of three equal-strength cavities whose centers lie not in the same line. By using the Riemann-Hilbert problem on a genus$(n-1)$ surface and the theory of abelian integrals we have also derived an integral representation in terms of hyperelliptic integrals for a $2 n$-parametric family (two of them are scaling parameters) of conformal mappings for the case $n \geq 4$ and $\omega(\infty)=\infty$, and the slits lie in the same line. We conjecture that (i) if the centers of equal-strength cavities lie in the same line and $\omega(\infty)=\infty$, then the function $\Psi(z)$ is free of poles and the solution exists when $\gamma<1$ and the function $\Psi(z)$ has $2 n$ poles in the domain $D^{e}$ when $\gamma>1$, and (ii) regardless of the value of the parameter $\gamma \neq 1$, if $\left|\zeta_{\infty}\right|<\infty$, there does not exist a set of $n \geq 4$ equal-strength cavities whose centers are not in the same line.

\section{Appendix: One cavity}

If $n=1$, then with no loss of generality $l_{0}$ and the point $\zeta_{\infty}$ may be selected as $[-1,1]$ and $\infty$, respectively. Such a map is defined up to one real parameter, and we assume that $\operatorname{Im} c_{-1}=0$. Denote $p_{1}(\zeta)=\zeta^{2}-1$. We fix the branch $f(\zeta)$ of $p^{1 / 2}(\zeta)$ in the $\zeta$-plane cut along $l_{0}$ by the condition $p_{1}(\zeta) \sim \zeta, \zeta \rightarrow \infty$. This branch is pure imaginary on the sides of the cut, $f(\xi \pm i 0)= \pm i|f(\xi)|$, $-1<\xi<1$, and real in the real axis outside the cut. We have

$$
F_{+}(\zeta)=\frac{A_{1}^{+}+A_{2}^{+} \zeta}{f(\zeta)}+i A_{0}^{+}, \quad F_{-}(\zeta)=\frac{i\left(A_{1}^{-}+A_{2}^{-} \zeta\right)}{f(\zeta)}+A_{0}^{-},
$$

where $A_{j}^{ \pm}(j=0,1,2)$ are arbitrary real constants. Due to the asymptotics $(2.12)$ of the functions $F_{ \pm}(\zeta)$ we have

$$
\begin{gathered}
A_{1}^{ \pm}=0, \quad A_{2}^{+}=c_{-1} \operatorname{Re}(b+\bar{a}), \quad A_{2}^{-}=c_{-1} \operatorname{Im}(b-\bar{a}), \\
A_{0}^{+}=c_{-1} \operatorname{Im}(b+\bar{a}), \quad A_{0}^{-}=c_{-1} \operatorname{Re}(b-\bar{a}) .
\end{gathered}
$$

Substituting these coefficients into (A.1) and then into (2.14) we derive

$$
\omega^{\prime}(\zeta)=\frac{c_{-1}}{2}\left[m_{-}+\frac{m_{+} \zeta}{f(\zeta)}\right], \quad m_{ \pm}=1 \pm \frac{\bar{b}}{\bar{a}}, \quad \psi(\zeta)=\bar{a} \frac{(a+b) \zeta-(a-b) f(\zeta)}{(\bar{a}+\bar{b}) \zeta+(\bar{a}-\bar{b}) f(\zeta)}
$$

Notice that

$$
\int_{l_{0}} \omega^{\prime}(\zeta) d \zeta=0
$$

and the map is one-to-one. The conformal map $z=\omega(\zeta)$ is defined up to an additive constant $B$, has the form 3 ]

$$
\omega(\zeta)=\frac{c_{-1}}{2}\left[m_{-} \zeta+m_{+}\left(\zeta^{2}-1\right)^{1 / 2}\right]+B,
$$

and $z=\omega(\zeta), \zeta \in l_{0}$, is a parametric equation of a family of ellipses $\left(c_{-1}\right.$ is an arbitrary nonzero real parameter). Denote $a_{1}+i b_{1}=\frac{1}{2} c_{-1} m_{-}, a_{2}+i b_{2}=\frac{1}{2} c_{-1} m_{+}$and put $B=0$. Then from (A.5) we may write

$$
x=a_{1} \xi \mp b_{2} \sqrt{1-\xi^{2}}, \quad y=b_{1} \xi \pm a_{2} \sqrt{1-\xi^{2}} .
$$

On excluding $\sqrt{1-\xi^{2}}$ we express $\xi$ through $x$ and $y$

$$
\xi=\frac{a_{2} x+b_{2} y}{a_{1} a_{2}+b_{1} b_{2}}
$$

We next square $x$ and $y$ in (A.6) and employ formula (A.7). After simple algebra we obtain a quadratic equation in $x$ and $y$

$$
\left(a_{2}^{2}+b_{1}^{2}\right) x^{2}+\left(a_{1}^{2}+b_{2}^{2}\right) y^{2}-2\left(a_{1} b_{1}-a_{2} b_{2}\right) x y=\left(a_{1} a_{2}+b_{1} b_{2}\right)^{2} .
$$


Since its discriminant $-4 \Delta=-4\left(a_{1} a_{2}+b_{1} b_{2}\right)^{2}$ is negative, equation (A.8) represents an ellipse.

Now, the function $\psi(\zeta)$ has to be analytic everywhere in $\mathbb{C} \backslash l_{0}$. Possible singularities of this function coincide with the zeros of the derivative of the map or, equivalently, with the zeros of the function $\eta(\zeta)=m_{-} \sqrt{\zeta^{2}-1}+m_{+} \zeta$. The number of zeros of $\eta(\zeta)$ inside the contour $\Gamma$ is given by

$$
Z=\frac{1}{2 \pi i} \int_{\Gamma} \frac{\eta^{\prime}(\zeta) d \zeta}{\eta(\zeta)}
$$

Computing the integral over the contour $\Gamma_{R}$, letting $R \rightarrow \infty$, and transforming the integral over the contour $l_{0}$ we obtain

$$
Z=1-\frac{m_{+} m_{-}}{\pi\left(m_{+}^{2}-m_{-}^{2}\right)} \int_{-1}^{1} \frac{d \xi}{\sqrt{1-\xi^{2}}\left(\xi^{2}+\mu^{2}\right)}, \quad \mu^{2}=\frac{m_{-}^{2}}{m_{+}^{2}-m_{-}^{2}} .
$$

This integral can be computed by making the subsequent substitutions $\xi=\cos \phi$ and $e^{i \theta}=w$ and applying the theory of residues in the $w$-plane. Eventually we derive that $Z=0$ if $|b / a|<1$ and $Z=2$ if $\gamma=|b / a|>1$. These poles can be easily determined

$$
\zeta_{1,2}= \pm \frac{i}{2}\left(\sqrt{\frac{\bar{b}}{\bar{a}}}-\sqrt{\frac{\bar{a}}{\bar{b}}}\right)
$$

In the case $\gamma=1$, the ellipse becomes a segment, while the poles (A.11) of the function $\omega^{\prime}(\zeta)$, $\zeta_{1,2}= \pm \sin \frac{1}{2}(\arg a-\arg b)$, lie in the segment $l_{0}$. Analysis of the second formula in (A.3) shows that they are removable singularities of the function $\psi(\zeta)$.

\section{References}

[1] Y.A. Antipov And V.V. Silvestrov, Method of Riemann surfaces in the study of supercavitating flow around two hydrofoils in a channel, Physica D, 235 (2007), pp. 72-81.

[2] N.V. BAnichuk, Problem of optimization of the shape of a hole in a plate under bending, Solid Mechanics - Izv. AN SSSR, Mekhanika Tverdogo Tela, no.3 (1977), pp. 81-88.

[3] G.P. Cherepanov, Inverse problems of the plane theory of elasticity, J. Appl. Math. Mech., 38 (1974), pp. 915-931.

[4] R. Courant, Dirichlet's Principle, Conformal Mapping, and Minimal Surfaces, Interscience Publishers, Inc., New York, N.Y., 1950.

[5] J.D. Eshelby, The determination of the elastic field of an ellipsoidal inclusion, and related problems, Proc. Roy. Soc. London A, 241 (1957), pp. 376-396.

[6] Y. Grabovsky and R.V. Kohn, Microstructures minimizing the energy of a two phase elastic composite in two space dimensions. II.: The Vigdergauz microstructure, J. Mech. Phys. Solids, 43 (1995), pp. 949- 972.

[7] H. KAnG, E. Kim AND G. W. Milton, Inclusion pairs satisfying Eshelby's uniformity property, SIAM, J. Appl. Math. 69 (2008), pp. 577-595.

[8] M.V. KeLDYsh, Conformal mappings of multiply connected domains on canonical domains, Uspekhi Matem. Nauk 6 (1939), pp. 90-119. 
[9] A.S. Kosmodamianskir, Stress state of anisotropic media with holes and cavities, Viszcza shkola, Kiev, 1976.

[10] N.I. Muskhelishvili, Some Basic Problems of the Mathematical Theory of Elasticity, P. Noordhoff, Ltd., Groningen, 1963.

[11] H. Neuber, Zur Optimierung der Spannungskonzentration, in Continuum Mechanics and Related Problems of Analysis, Nauka, Moscow (1972), pp. 375-380.

[12] C.-Q. Ru And P. Schiavone, On the elliptic inclusion in anti-plane shear, Math. Mech. Solids, 1 (1996), pp. 327-333.

[13] G.N. SAvin, Stress Distribution Around Holes, Naukova Dumka, Kiev, 1968 (NASA Tech. Trans., Washington D.C., 1970).

[14] G.P. SendeckyJ, Elastic inclusion problems in plane elastostatics, Int. J. Solids Structures 6 (1970), pp. 1535-1543.

[15] R.-J. Shin AND L.T. Wheeler, Two-dimensional inhomogeneities of minimum stress concentration, Quart. Appl. Math. 64 (1986), pp. 567-582.

[16] G. Springer, Introduction to Riemann Surfaces, Addison-Wesley, Reading, MA, 1956.

[17] S.B. Vigdergauz, Integral equation of the inverse problem of the plane theory of elasticity, J. Appl. Math. Mech., 40 (1976), pp. 518-522.

[18] S.B. Vigdergauz, Effective elastic parameters of a plate with a regular system of equalstrength holes, Solid Mechanics - Izv. AN SSSR, Mekhanika Tverdogo Tela, 21, no.2 (1986), pp.165-169.

[19] S.B. Vigdergauz, Constant-stress inclusions in an elastic plate, Math. Mech. Solids, 5 (2000), pp. 265-279.

[20] S.B. Vigdergauz, Stress smoothing holes in planar domains, J. Mech. Materials Structures, 5 (2010), pp. 987-1006. 